\title{
Review
}

\section{Loss-of-Function Rodent Models for Parkin and PINK1}

\author{
Marusela Oliveras-Salvá ${ }^{1}$, Anne-Sophie Van Rompuy ${ }^{1}$, Bavo Heeman, Chris Van den Haute and \\ Veerle Baekelandt* \\ Laboratory for Neurobiology and Gene Therapy, Division of Molecular Medicine, Katholieke Universiteit Leuven, \\ Leuven, Belgium
}

\begin{abstract}
Parkinson's disease is a common neurodegenerative disorder whose aetiology is not yet fully understood. In the past ten years, the discovery of genes linked to hereditary forms of the disease has impelled the development of animal models. These should lead to the identification of novel pathways that provide insight into the functionality of the proteins involved and the pathogenesis of the sporadic forms of the disease. In particular, loss-of-function mutations in the parkin and PINK1 (phosphatase and tensin homolog (PTEN)-induced kinase 1) genes account for most of the cases of familial autosomal recessive parkinsonism. Both parkin and PINK1 knockout rodent models are now available, which display an overall mild phenotype consisting of a mitochondrial dysfunction together with changes in dopamine metabolism and oxidative stress. However, up till now these models fail to reproduce the main hallmarks of Parkinson's disease: the dopaminergic cell loss in the substantia nigra and the presence of cytoplasmic inclusions, named Lewy bodies, in the remaining dopaminergic neurons. We here review the most important knockout and knockdown rodent models generated so far for these two recessive Parkinson's disease-causing genes. We critically feature their main characteristics and their impact on the research field, and propose some future directions for the study and modelling of the loss of function of parkin and PINK1 in rodents.
\end{abstract}

Keywords: Parkinson's disease, parkin, PINK1, model, knockout, knockdown, mice, mitochondria

\section{INTRODUCTION}

Parkinson's disease (PD) is the most common neurodegenerative movement disorder. It affects more than $1 \%$ of the population aged over 65 years old and has a mean age of onset between 60 and 70 years old, although 5 to $10 \%$ of the PD patients are younger than 50 years old [1]. Currently, the number of patients suffering from the disease is 4.6 million people worldwide and it is expected to double in the coming thirty years [2]. Clinically, the diagnosis of PD is based on the presence of symptoms such as bradykinesia,

\footnotetext{
${ }^{1}$ These authors contributed equally to this work.

${ }^{*}$ Correspondence to: Veerle Baekelandt, PhD, Laboratory for Neurobiology and Gene Therapy, Katholieke Universiteit Leuven, Kapucijnenvoer 33, box 7001, 3000 Leuven, Belgium. Tel.: +32 16336332; Fax: +32 16336336; E-mail: Veerle.Baekelandt@med. kuleuven.be.
}

cogwheel rigidity, resting tremor and postural instability. The neuropathology observed post-mortem in these patients comprises both the neurodegeneration in a specific region of the brain involved in motor control, the substantia nigra (SN) pars compacta, and the prevalence of proteinaceous cytoplasmic inclusions called Lewy bodies (LBs). However, other brain regions are also affected during the progression of the disease, which probably account for other non-motor PD symptoms such as sleep disturbance, depression and psychosis [3, 4].

As neurodegenerative disorders are predominantly sporadic, the hereditary component of PD was long denied. However, in 1997 Polymeropoulos et al. documented the presence of a missense mutation in the SNCA gene as a cause of autosomal dominant familial PD [5]. Subsequently, the possible link between sporadic and hereditary PD forms was strengthened 
by the discovery of $\alpha$-synuclein as the main component of LBs in sporadic cases of PD [6]. Over the past decade, research on PD has increased tremendously by the identification of more genes that are linked to rare familial forms of the disease. These inherited forms account for 5 to $10 \%$ of the total number of PD patients and cause in most cases early-onset $(<50$ years old at onset) disease. Whole locus multiplications in the SNCA gene and mutations in the leucine-rich repeat kinase 2 (LRRK2) gene account for most of the cases of autosomal dominant familial PD. Furthermore, lossof-function mutations in the parkin, PINK1 and DJ-1 genes lead to hereditary autosomal recessive juvenile PD (AR-JPD). Other minority genes, whose link to $\mathrm{PD}$ is still confusing, have recently been reported as a result of genome-wide association studies [7, 8].

The molecular aetiology of PD remains unclear. Protein misfolding and proteasomal deficiency, as well as mitochondrial dysfunction and oxidative stress, are thought to be the main phenomena leading to dopaminergic cell death in the SN. However, it is not yet fully known whether all these different pathways and proteins related to PD interplay, although several lines of evidence suggest a similar mechanistic route leading to PD in both idiopathic and familial forms of the disease. For this reason, it is relevant to deep into the molecular basis of the familial forms in order to better understand the pathogenesis of the more common sporadic forms of the disease. Parkin was very early on identified as an E3-ubiquitin ligase which led to the initial hypothesis that parkin played a role in proteasomal degradation of several target proteins, thereby reducing unfolded protein stress-induced cell death $[9,10]$. However, more recent findings have highlighted a role for parkin, in combination with PINK1, in mitophagy, where mitochondrial integrity is maintained through selective autophagic clearance of damaged mitochondria $[11,12]$. PINK1 is a mitochondrial kinase and most pathogenic mutations in the PINK1 gene have been shown to cause down-regulation of its kinase activity [13].

The two PD-associated proteins appear to play a crucial role in mitophagy, an organellar quality control to ensure the mitochondrial structural unity (reviewed in [14-17]). Loss of mitochondrial membrane potential results in stabilization of the PINK1 protein, selective accumulation in depolarized mitochondria and translocation of parkin from the cytosol to the mitochondria. PD-associated parkin and PINK1 mutations have been demonstrated to abrogate this mitophagic pathway at distinct steps [18]. The translocation of parkin by PINK1 to these damaged mitochondria has recently been shown to activate the ubiquitin-proteasome system (UPS) resulting in widespread degradation of mitochondrial surface proteins, prior to autophagic clearance, thereby providing a causal and temporal link between the UPS and mitophagy [19-21].

However, many questions remain unanswered in the field such as the exact mechanism leading to PINK1 accumulation upon depolarization, the characteristics of the PINK1/parkin interaction and the implications of mitophagy in neurodegeneration. Hence, the development of animal models that recapitulate the pathological features present in human patients is now of uttermost importance to gain insight into the functionality of the proteins involved and the pathogenesis of idiopathic PD. Up till now, there have been several attempts to generate PD animal models but, somewhat surprisingly, most of these models have failed to display the main hallmarks of the disease. We here review the most relevant rodent models developed so far for parkin and PINK1 null mutations and their relevance for the understanding of PD's neurodegenerative mechanisms.

\section{PARKIN}

The parkin gene was the second gene to be linked to PD after the SNCA gene. It corresponds to the PARK2 locus that was mapped to chromosome 6q25.2-27 in 1997 [22]. One year later, Kitada et al. identified the gene and named it parkin, which is encoded by 12 exons spanning $1.53 \mathrm{Mb}$ [23]. It is expressed in various human tissues, including brain, heart, testis and skeletal muscle. In the brain, parkin is present in many regions, including the $\mathrm{SN}$. The parkin protein has 465 amino acids with a predicted molecular weight of $52 \mathrm{kDa}$ and functions as an E3-ubiquitin ligase. It contains an $\mathrm{N}$-terminal ubiquitin-like domain and a C-terminal end characterized by two $\mathrm{Zn}^{2+}$-binding RING (really interesting new gene) domains separated by an In-Between-RING domain [9]. Recently, a novel $\mathrm{Zn}^{2+}$-binding domain was discovered upstream of the RING1 domain, named RING0 [24].

An estimated $50 \%$ frequency of loss-of-function mutations in parkin has been reported in AR-JPD whereas their prevalence in early-onset PD forms without clear family history is around $15 \%[25,26]$. The first mutations found in the parkin gene were described in Japanese patients carrying vast homozygous deletions of exons 3 to 7 or exon 4 alone [23]. In the last decade, a large variety of mutations has been identified throughout all the domains of parkin. These mutations 
include deletions and multiplications of one or several exons, as well as point mutations, missense and nonsense mutations, and splice site mutations [27]. Data on neuropathological studies of patients with AR-JPD due to parkin mutations are limited and conflicting. In all cases, there is a loss of pigmented neurons in the $\mathrm{SN}$ and in the locus coeruleus, but most described cases lack $\alpha$-synuclein-positive inclusions in the $\mathrm{SN}$ [28-33]. Only in a few cases LBs were found [34, 35]. In one patient, LB-like inclusions were found in the lumbar spinal motor neurons, but not in the SN [36]. The reason for this scarce synucleinopathy in parkin patients has so far not been resolved.

\section{Generation of parkin loss-of-function rodents}

Knocking out the function of parkin in mice has been accomplished by genetic deletion of exon 2, exon 3 or exon 7, all resulting in the absence of parkin protein expression (Table 1). The different knockout mice were created using a similar strategy for the different strains: the targeted gene region was replaced by a selection cassette through homologous recombination in embryonic stem cells.

Exon 2 was chosen as a target because it encodes most of the functionally important ubiquitin-like domain and mutations in this exon have been reported in patients with AR-JPD [27, 37]. Two mouse lines with a deletion of exon 2 were described [37, 38]. Perez et al. generated a mouse strain on a mixed C57BL6/129S4 genetic background and, in parallel, a coisogenic mutated mouse line was created on a 129S4 genetic background [37]. Sato et al. injected TT2 embryonic stem cells carrying the desired homologous recombination into $\mathrm{C} 57 \mathrm{BL} / 6 \mathrm{~J}$ blastocysts and chimeric offspring was further crossed with C57BL/6J mice [38].

Different groups generated parkin-deficient mouse lines with a deletion of exon 3 since deletion mutations in this exon are common in AR-JPD [39-44]. Itier et al. established breeding colonies on a 129Sv background and on a 50/50\% mixed 129SV/C57BL6 background [39] whereas two other groups generated mice lacking exon 3 on a mixed C57BL6/129SV background of unspecified proportions [40, 41]. Zhu et al. described parkin null mice with a mixed genetic background of 94\% C57BL6 and 6\% 129SvJ [42].

Exon 7 encodes the first RING finger domain of parkin, a region where several disease-associated point mutations are clustered [40, 44]. Only one group reported a mouse line with a targeted disruption of exon 7 [45]. The deletion was established by introduction of a targeting cassette containing exon 7 flanked by LoxP sites and subsequent Cre-mediated recombination. No information on the primary background of these mice was provided.

Very recently, the same group reported the generation of a conditional parkin knockout mouse using a combination of transgenic and viral vector technology [46]. Stereotactic injection of a Green Fluorescent Protein (GFP)-fused Cre recombinase expressing lentiviral vector (Lenti-GFPCre) in the SN of 6-8 weeks old mice in which exon 7 was flanked by loxP sites (parkin ${ }^{\mathrm{Flx} / \mathrm{Flx}}$ ) resulted in the deletion of exon 7 and in the absence of parkin protein in the ventral midbrain. Parkin ${ }^{\mathrm{Flx} / \mathrm{Flx}}$ animals injected with a lentiviral vector expressing GFP (Lenti-GFP) were used as a control.

The quaking viable $\left(\mathrm{qk}^{\mathrm{v}}\right)$ mouse has been known for almost five decades and has been studied as a model for demyelination of the central nervous system [47]. These mice have a large spontaneous deletion of approximately $1 \mathrm{Mb}$ in chromosome 17 , which leads to tremor and tonic-clonic seizures in homozygotes. Recently, it was discovered that the parkin gene and the parkin co-regulated gene lie within this deletion, resulting in a lack of parkin protein expression $[48,49]$.

\section{General health, neuroanatomy and morphology}

Overall, no difference in general appearance, morbidity or mortality was observed between wild-type and parkin-deficient mice up to 24 months of age. However, one group reported an increase in mortality compared to age-matched control littermates [50]. In addition, these aged mice showed abnormal postures, with a dorsal kyphosis (outward curvature of the thoracic spine) and focal hair loss.

Three exon 3-deleted mouse lines displayed a significantly reduced body weight $[39,51,52]$, that was concomitant with a decreased body temperature in one of these studies [39]. In addition, the average body weight of knockout mice with a mixed C57BL6/129S4 background described by Perez et al. was decreased compared to controls at 6 months of age but not younger or older [37]. However, this was not the case for mice with a 129S4 genetic background generated by the same group.

Gross examination of the brains of the different knockout mice revealed no major changes in brain morphology, weight and size. The majority of the parkin null mice presented a normal structure of the dopaminergic neurons in the SN. No loss of tyrosine hydroxylase (TH)-positive neurons in the $\mathrm{SN}$ or in their 
Table 1

Key features of parkin knockout and Q311X transgenic mouse models

\begin{tabular}{|c|c|c|c|c|c|c|c|c|c|}
\hline Characteristics & $\begin{array}{l}\text { Itier et al., } \\
2003\end{array}$ & $\begin{array}{l}\text { Goldberg et al., } \\
\text { 2003; Palacino } \\
\text { et al, 2004; } \\
\text { Kitada et al., } \\
2009\end{array}$ & $\begin{array}{l}\text { von Coelln } \\
\text { et al., } 2004\end{array}$ & $\begin{array}{l}\text { Perez et al., } \\
\text { 2005a, 2005b }\end{array}$ & $\begin{array}{l}\text { Sato et al., } \\
2006\end{array}$ & $\begin{array}{l}\text { Kitao et al., } \\
2006 ; \\
\text { Oyama et al., } \\
2010\end{array}$ & $\begin{array}{l}\text { Zhu et al., } \\
2007 ; \\
\text { Stichel et al., } \\
2007\end{array}$ & $\begin{array}{l}\text { Rodriguez- } \\
\text { Navarro et al., } \\
2007\end{array}$ & $\begin{array}{l}\text { Lu et al., } \\
2009\end{array}$ \\
\hline \multicolumn{10}{|l|}{ Development of the model } \\
\hline Exon targeted & 3 & 3 & 7 & 2 & 2 & 3 & 3 & cfr. Itier et al. & Q311X \\
\hline \multicolumn{10}{|l|}{ General } \\
\hline Mortality risk & $=$ & $=$ & $=$ & $=$ & & $=$ & $=$ & $\uparrow$ & \\
\hline Body weight & $\downarrow$ & $\downarrow$ gain & $=$ & $\downarrow(6 \mathrm{~m})$ & & $\downarrow$ & $=$ & & \\
\hline Body temperature & $\downarrow$ & & & $=$ & & & & & \\
\hline \multicolumn{10}{|l|}{$\begin{array}{l}\text { DAergic neurons \& DA } \\
\text { metabolism }\end{array}$} \\
\hline Number in SN & $=$ & $=$ & $=$ & $=$ & $=$ & $=$ & $=$ & $\downarrow(24 \mathrm{~m})$ & $\downarrow(16 \mathrm{~m})$ \\
\hline DA levels striatum & $=(11 \mathrm{~m})$ & $=(6-24 \mathrm{~m})$ & $=(18 \mathrm{~m})$ & $=(22 \mathrm{~m})$ & $=(12 \mathrm{~m})$ & $=(3-12 \mathrm{~m})$ & $=$ & & $\downarrow(19-21 \mathrm{~m})$ \\
\hline DA levels other regions & $\uparrow \operatorname{limbic}(11 \mathrm{~m})$ & & & & $\uparrow \mathrm{SN}(12 \mathrm{~m})$ & & & & \\
\hline DA uptake & $\downarrow^{\dagger}$ & $=(2-3 \mathrm{~m})$ & & & & & $=(3 \mathrm{~m})$ & & \\
\hline DA release & $\downarrow^{\dagger}$ & $\downarrow(2-3 \mathrm{~m})$ & & & $\downarrow(12 \mathrm{~m})$ & $\downarrow(3-12 \mathrm{~m})$ & & & \\
\hline DA synthesis & & & & & $\downarrow(12 \mathrm{~m})$ & & & & \\
\hline DOPAC levels & $\uparrow(11 \mathrm{~m})$ & $=(6-24 \mathrm{~m})$ & $=(18 \mathrm{~m})$ & $=(22 \mathrm{~m})$ & $=(12 \mathrm{~m})$ & $=(3-12 \mathrm{~m})$ & $\downarrow$ & & $\downarrow(19-21 \mathrm{~m})$ \\
\hline HVA levels & & $=(6-24 \mathrm{~m})$ & $=(18 \mathrm{~m})$ & $=(22 \mathrm{~m})$ & $=(12 \mathrm{~m})$ & $=(3-12 \mathrm{~m})$ & $\uparrow$ & & \\
\hline \multicolumn{10}{|l|}{ Other neuropathology } \\
\hline Glutamate release & $\downarrow(14-15 \mathrm{~m})$ & & & & & & & & \\
\hline Glutathione levels & $\uparrow\left({ }^{\dagger}\right.$ and $\left.11 \mathrm{~m}\right)$ & & & & & & & $\uparrow(2 \mathrm{~m}), \downarrow(24 \mathrm{~m})$ & \\
\hline Number NA neurons & & $=$ & $\downarrow(2-18 \mathrm{~m})$ & & $=$ & & $=$ & & \\
\hline NA levels & $=(11 \mathrm{~m})$ & & $\begin{array}{l}\downarrow(18 \mathrm{~m}) \mathrm{OB}, \mathrm{sp} \\
\text { cord }\end{array}$ & $=(22 \mathrm{~m})$ & & & $=$ & & \\
\hline 5-HT metabolism & $=(11 \mathrm{~m})$ & & & & & $=(3-12 \mathrm{~m})$ & $=$ & & \\
\hline \multicolumn{10}{|l|}{ Mitochondria } \\
\hline Morphology & & $=$ & & & & & $\uparrow *(12-14 \mathrm{~m})$ & & \\
\hline Size & & $=$ & & & & & & & \\
\hline Number & & $=$ & & & & & $=(12-14 \mathrm{~m})$ & & \\
\hline Respiration deficiency & & st. $3+4$ & & & & & st. $3 *(14 \mathrm{~m})$ & & \\
\hline Oxidative stress & & $\uparrow(18-20 \mathrm{~m})$ & & $=(22 \mathrm{~m})$ & & & & & \\
\hline \multicolumn{10}{|l|}{ Neurotoxin vulnerability } \\
\hline MPTP & & & $\begin{array}{l}=(2 ; 3 ; 12 \mathrm{~m}) \text { in } \\
{[63]}\end{array}$ & & & & $=(18 \mathrm{~m})$ & & \\
\hline DSP-4 & & & $=(18 \mathrm{~m})$ in $[63]$ & & & & & & \\
\hline Rotenone & $\uparrow^{\dagger}$ in [64] & & & & & & & & \\
\hline Methamphetamine & & & & $=(3 \mathrm{~m})$ & & & & & \\
\hline 6-OHDA & & & & $=(3 \mathrm{~m})$ & & & & & \\
\hline
\end{tabular}


Table 1

\begin{tabular}{|c|c|c|c|c|c|c|c|c|c|}
\hline Characteristics & $\begin{array}{l}\text { Itier et al., } \\
2003\end{array}$ & $\begin{array}{l}\text { Goldberg et al., } \\
\text { 2003; Palacino } \\
\text { et al, 2004; } \\
\text { Kitada et al., } \\
2009\end{array}$ & $\begin{array}{l}\text { von Coelln } \\
\text { et al., } 2004\end{array}$ & $\begin{array}{l}\text { Perez et al., } \\
\text { 2005a, 2005b }\end{array}$ & $\begin{array}{l}\text { Sato et al., } \\
2006\end{array}$ & $\begin{array}{l}\text { Kitao et al., } \\
2006 \text {; } \\
\text { Oyama et al., } \\
2010\end{array}$ & $\begin{array}{l}\text { Zhu et al., } \\
2007 ; \\
\text { Stichel et al., } \\
\text { 2007; }\end{array}$ & $\begin{array}{l}\text { Rodriguez- } \\
\text { Navarro et al., } \\
2007\end{array}$ & $\begin{array}{l}\text { Lu et al., } \\
2009\end{array}$ \\
\hline \multicolumn{10}{|l|}{ Locomotor activity } \\
\hline Open field test & $\downarrow(6 \mathrm{~m})$ & $=(6-18 \mathrm{~m})$ & $=(18 \mathrm{~m})$ & $\begin{array}{l}\uparrow(12 \mathrm{~m}),= \\
(3 ; 6 ; 18-22 \mathrm{~m})\end{array}$ & $=(12 \mathrm{~m})$ & & $=(6-21 \mathrm{~m})$ & & $\begin{array}{l}\downarrow(16-21 \mathrm{~m})= \\
(6-12 \mathrm{~m})\end{array}$ \\
\hline Balance beam & & $\begin{array}{l}\downarrow(2-4 ; 7 ; \\
18 \mathrm{~m})\end{array}$ & & $=(19 \mathrm{~m})$ & & & & & $\downarrow(16-19 \mathrm{~m})$ \\
\hline Rotarod & & $=(6-18 \mathrm{~m})$ & $=(3-24 \mathrm{~m})$ & $\begin{array}{l}\uparrow(6 \mathrm{~m}),= \\
(3 ; 12 ; 18 \mathrm{~m})\end{array}$ & $=(12 \mathrm{~m})$ & $=(3-12 \mathrm{~m})$ & $=(6 \mathrm{~m})$ & & \\
\hline Amphetamine response & $\downarrow(6 \mathrm{~m})$ & & & $=(3 \mathrm{~m})$ & & & & & \\
\hline Footprint analysis & & & & $=(19 \mathrm{~m})$ & & & & $\downarrow(18 \mathrm{~m})$ & \\
\hline Negative-geotaxis test & & & & $\begin{array}{l}\downarrow(18 \mathrm{~m}),=(6 ; \\
12 \mathrm{~m})^{\$}\end{array}$ & & & & & \\
\hline Catalepsy test & & & & $=(6 ; 12 ; 19 \mathrm{~m})$ & & & & & \\
\hline \multicolumn{10}{|l|}{ Learning and memory } \\
\hline T-maze & $\downarrow(4 \mathrm{~m})$ & & & $=(12 ; 20 \mathrm{~m})$ & & & & & \\
\hline Morris water maze & & & & $=(20 \mathrm{~m})$ & & & $\downarrow$ & & \\
\hline \multicolumn{10}{|l|}{ Somatosensory function } \\
\hline Adhesive removal & & $\begin{array}{l}\downarrow(2-4 ; \\
7 \mathrm{~m})=(18 \mathrm{~m})\end{array}$ & & $=(19 \mathrm{~m})$ & & & & & $\begin{array}{l}\downarrow(16-19 \mathrm{~m}),= \\
(3-12 \mathrm{~m})\end{array}$ \\
\hline Acoustic startle & & & $\downarrow(9 \mathrm{~m})$ & $\uparrow(12 \mathrm{~m})^{\$}$ & & & & & \\
\hline Tail-flick & & & & $=(21 \mathrm{~m})$ & & & & & \\
\hline \multicolumn{10}{|l|}{ Emotionality } \\
\hline Light/dark transition & & & & $=(12 ; 21 \mathrm{~m})$ & & & $\downarrow(6-15 \mathrm{~m})$ & & \\
\hline Elevated plus maze & & & & $=(19 \mathrm{~m})$ & & & & & \\
\hline Forced-swim & & & & $=(12 ; 20 \mathrm{~m})$ & & & & & \\
\hline Tail suspension & & & & $=(20 \mathrm{~m})$ & & & & & \\
\hline Parkin substrate levels & & $=$ & $\begin{array}{l}\uparrow \mathrm{p} 38(18 \mathrm{~m}) \text { in } \\
{[70], \uparrow \text { PARIS }} \\
(18-24 \mathrm{~m}) \text { in } \\
{[46]}\end{array}$ & & & & & & $\begin{array}{l}\uparrow \text { PK-resistant } \\
\alpha \text {-synuclein } \\
(16 \mathrm{~m})\end{array}$ \\
\hline
\end{tabular}

$\uparrow$ : increase, $\downarrow$ : decrease,=: no changes (in comparison to control); DA: dopamine; SN: substantia nigra; DOPAC: dihydroxyphenylacetic acid; HVA: homovanillic acid; NA: noradrenaline; OB: olfactory bulb, sp cord: spinal cord; 5-HT: 5-hydroxytryptamine; st: state; MPTP: 1-methyl-4-phenyl-1,2,3,6-tetrahydropyridine; DSP-4: $N$-(2-chloroethyl)- $N$-ethyl-2-bromobenzylamine; 6-OHDA: 6-hydroxydopamine; PK: proteinase $\mathrm{K} ;{ }^{\dagger}$ : in fetal midbrain neuronal cultures; *: findings in double mutant parkin knockout/ $\alpha$-synuclein transgenic mice; $\$$ : findings not reproducible on a 129S4 genetic background; mice ages are indicated in brackets if available, $\mathrm{m}$ : month(s). 
striatal projections was detected up to 24 months of age. Surprisingly, in 2007 one group reported a $35 \%$ loss of TH-positive neurons in the SN of 24-monthold knockout mice [50]. Those parkin null mice were created by intercross of heterozygous exon- 3 deleted mice generated by Itier et al. [39]. However, this finding was not reported in the first publication of Itier and colleagues in 2003 and has not been reproduced in later publications.

The number and anatomy of the noradrenergic neurons in the locus coeruleus appeared normal in the exon 3- and exon 2-deleted mouse lines, contrary to the exon 7-deleted line where mice showed a reduction of approximately $20 \%$ in the number of $\mathrm{TH}-$ immunoreactive neurons in the locus coeruleus. This loss of neurons was noticed in both young ( 2 months old) and old (12-18 months old) animals and did not appear progressive. However, this phenotype was not present in the whole population: around $30 \%$ of the parkin null mice had the same number of TH-positive neurons in the $\mathrm{SN}$ as the wild-type mice. Furthermore, neurochemical analysis revealed a reduced concentration of noradrenaline in the olfactory bulb and the spinal cord, two regions that are innervated by noradrenergic neurons in the locus coeruleus [45].

Interestingly, the conditional knockout of parkin in adult animals led to approximately $40 \%$ loss of $\mathrm{TH}-$ positive neurons in the $\mathrm{SN}$ at 10 months after the stereotactic injection of Lenti-GFPCre in parkin ${ }^{F l x} / F l x$ mice compared to control mice injected with LentiGFP. Since there was no loss of dopaminergic neurons after 3 months, this loss was reported to be progressive [46]. The most likely explanation for this loss of dopaminergic neurons by adult local knockout of parkin is the avoidance of potential compensatory mechanisms occurring in traditional transgenesis. Of course, the effect of a second stressor due to the use of viral vectors or to overexpression of the C-protein cannot be completely ruled out, although the fact that the dopaminergic cell loss could be rescued by coinjection of a second lentiviral vector pleads against this.

\section{Neurotransmission systems and metabolism}

Very subtle changes in the nigrostriatal dopamine neurotransmission system and dopamine metabolism have been shown in parkin-deficient mice, but discrepancies between the different mouse lines make it difficult to draw conclusions.

High-performance liquid chromatography quantification of striatal tissue of the different mouse lines revealed normal levels of dopamine in parkin knockout mice aged 6 to 24 months. However, an increase in dopamine levels in the $\mathrm{SN}$ was reported in mice at 12 months old [38]. Furthermore, Itier and colleagues showed a similar increase in the limbic regions of 11month-old knockout mice. The striatal levels of the major dopamine metabolites, dihydroxyphenylacetic acid (DOPAC) and homovanillic acid (HVA), have repeatedly been analyzed by different research groups. In general, no differences were detected between parkin knockout mice and wild-type mice. Yet, Zhu et al. reported a significant increase of HVA levels and slightly, but not statistically significant, reduced levels of DOPAC in the striatum of parkin knockout mice, resulting in an increased striatal HVA/DOPAC ratio [42]. Itier et al. revealed an increase in DOPAC levels in the limbic regions of knockout mice [39]. The same mice presented increased ratios of DOPAC/DA and DOPAC/HVA in the brain stem. In addition, the ratio of DOPAC/3- methoxytyramine (3-MT) was increased in the striatum, the diencephalon and the limbic regions [39]. This is in contrast with the finding of another group that reported an unchanged DOPAC/3-MT ratio in the striatum [37]. Von Coelln et al. described an unchanged ratio of DOPAC and HVA to dopamine [45]. Overall, data about dopamine metabolism are contradictory between the different mouse lines and difficult to interpret. This variability in results may be attributed to discrepancies in measuring methods or to small sample size.

Another interesting result is the decrease of dopamine transporter and vesicular monoamine transporter 2 levels in the striatum of 15-month-old knockout mice measured by western blot. In addition, the dopamine uptake and the amphetamine-induced dopamine release, which is an index of newly synthesized intracytoplasmic neurotransmitter release, were decreased in fetal midbrain neuronal-enriched cultures. However, basal release and $K^{+}$-evoked release of dopamine, an index of vesicular dopamine release, were unchanged [39]. Goldberg and colleagues reported elevated levels of extracellular dopamine in the striatum [40]. The synaptic excitability of striatal medium-sized spiny neurons, measured on corticostriatal brain slices, was decreased in these animals. Furthermore, the levels and the binding affinities of D1 and D2 receptors in the striatum were investigated, but no significant differences were detected in these mice $[40,53]$. This is in contrast with the results of an ex vivo autoradiography study of the striatum of 12month-old parkin knockout mice, where an increase in receptor binding levels of both D1 and D2 receptors 
was observed. In addition, synthesis of dopamine was decreased and dopamine release after treatment with metamphetamine (METH) was reduced in these mice [38]. By means of in vivo voltammetry, a decrease in the amplitude of evoked extracellular dopamine overflow was found in the striatum of parkin knockout mice at 3 and 6 months old when compared to wild-type littermates [52]. Likewise, the evoked dopamine release in acute striatal slices of parkin knockout mice was similarly decreased when measured by amperometric recordings [53].

Other neurotransmission systems have been studied in the parkin-deficient mice. In none of the different exon 3-deleted mouse lines arguments were found for an altered 5-hydroxytryptamine metabolism [39, 42, 52]. This was not investigated in any of the other mouse lines. Examination of the glutamatergic synaptic transmission in the hippocampus of parkin knockout mice revealed a decrease in presynaptic glutamate release [39].

As for the $\mathrm{qk}^{\mathrm{v}}$ mice, alterations in dopamine metabolism have also been reported such as increased levels of HVA and DOPAC in the striatum and the nucleus accumbens with olfactory tubercles, respectively [54]. However, the levels of dopamine were unaltered. The density of dopamine D2 receptors in the striatum was increased, but the receptor affinity for the $[3 \mathrm{H}]$-spiperone radioligand was reduced. Behaviourally, these mice were more sensitive to stimulation with D1 receptor agonists and displayed a decreased sensitivity to D2 receptor stimulation.

In conclusion, although these results are partially inconsistent, they suggest that parkin plays a role in dopamine neurotransmission. The most common finding is a decrease in dopamine release in parkin knockout mice. Finally, some results might indicate a possible function for parkin in glutamate transmission.

\section{Behaviour}

A whole battery of behavioural tests has been applied on the different parkin null mice. Unfortunately, they have been tested in various sets of behavioural tests, so it is difficult to compare the separate mouse lines. The tests can mostly be divided into the following categories: somatosensory function, motor function, emotionality and learning and memory.

Somatosensory function was tested with the adhesive removal test, the tail-flick test and the response to acoustic stimuli. Parkin knockout mice of one exon 3-deleted line had more difficulties to remove small adhesive labels fixed to their forehead when aged 2-4 and 7 months old but not at 18 months old [40]. Both the adhesive removal test and the tail-flick test were performed in an exon 2-deleted mouse line, but no differences were found between knockout and wild-type mice [37]. Von Coelln et al. noticed a reduced acousticstartle response in 9-month-old exon 7-deleted mice. Since the mice responded to the softest startle tones, the reduction in startle response was not attributed to a hearing deficit but rather to the loss of noradrenergic transmission secondary to the reduced number of noradrenergic neurons in the locus coeruleus of these mice [45]. In contrast, exon 2-deleted mice on a mixed C57BL6/129S4 background exhibited an increased sensitivity to the acoustic-startle response at 12 months of age. The same test was repeated with knockout and wild-type mice on a coisogenic 129S4 strain and the results could not be confirmed [37].

In general, no significant alteration in locomotor activity was reported in parkin knockout mice. A single group observed a reduced spontaneous gross locomotor activity in 6-month-old parkin null mice [39]. In these mice, administration of amphetamine (1-5 mg/kg) did not increase motor activation whereas wild-type mice became hyperactive. In contrast, another study showed no difference in locomotor response in 3-month-old knockout mice compared to controls after treatment with $4 \mathrm{mg} / \mathrm{kg}$ of amphetamine [37]. In addition, no differences were observed in these mice when aged 19 months in the balance beam task and footprint gait analysis. However, Goldberg et al. reported an increased number of slips and slips per step in their knockout mice at all ages in a similar beam traversal task [40]. As for the footprint analysis, a different study reported a shortening of the stride of hind limb in aged parkin knockout mice compared to wild-type animals [50]. In the rotarod test, no striking differences were detected between the different parkin knockout mice up to 24 months of age $[37,38,40,42,45,52]$. The negative-geotaxis test was performed in one group to identify deficits in motor coordination. In this test, mice were placed on a vertical wire grid in a head-down position and the time to orient their head into a head-up position was recorded. Interestingly, parkin knockout mice on a mixed C57BL6/129S4 background took longer to orient themselves to a head-up position at 18 months old, but not at 6 or 12 months old. Unfortunately, this could not be repeated on a 129S4 genetic background [37].

Psychiatric complications such as anxiety have been associated with idiopathic PD patients [55]. Some evidence was found for increased anxiety in exon 
3-deleted parkin knockout mice [42]. Indeed, these mice displayed a decreased exploratory behaviour and increased thigmotaxic behaviour in the open field test compared to controls. In the light/dark transition test, 6- and 15-month-old knockout mice spent less time in the illuminated compartment and made less transitions between the light/dark compartments [42]. In contrast, another study found no evidence of increased anxiety in the elevated-plus maze test in 19-month-old exon 2-deleted mice and no differences were detected either in the light-dark exploration test at 12 and 20 months of age when compared to wild-type mice [37].

Some deficits in spatial learning and memory in parkin knockout mice were reported as assessed by the Morris water maze test [42]. These mice exhibited slightly longer latencies to find the fixed hidden platform during the 7-day training period. On the 8th day, when the platform was removed, the knockout mice made less crossings in the former platform quadrant than the control mice. Remarkably, these mice also displayed a thigmotaxic behaviour in the water tank, with a preference to swim in the periphery of the water tank [42]. In contrast to the findings mentioned above, Perez et al. found no disabilities in learning and memory, neither in the Morris water maze nor in the T-maze spontaneous alternation task [37]. However, another group reported a decreased spontaneous alternation in the exploration of the unknown arm of a T-maze in 4month-old parkin knockout mice, indicating that these mice are probably less able to remember what they have already explored [39]. Intriguingly, during a second exposure to the hanging thread test 3 months after the first test where no difference was shown between parkin knockout and wild-type mice, the number of control mice rejecting the challenge was larger than the number of knockout mice. This was also explained by a learning deficit in the parkin-deficient mice [39].

In conclusion, the behavioural performance of the distinct parkin knockout mice showed several discrepancies. This may be caused by technical and experimental differences among the researchers or by the diverse types of parkin mutations and targeting strategies used to develop the parkin null mice. It is also known that the genetic background of the mouse strain influences the outcome of the experiments. For instance, a wide range of responses in the acoustic-startle test has been reported between different inbred mouse strains [56]. Perez et al. performed the most extensive behavioural analysis and subtle deficits were reported in different tests using mice on a mixed C57BL6/129S4 background. However, most of the observed differences could not be reproduced when using coisogenic mice on a 129S4 background [37]. No other groups studied the effect of different genetic backgrounds in such a systematic way. In addition, most of the behavioural tests were performed in both young and old mice and no clear age-dependent changes could be revealed.

\section{Mitochondrial morphology and function}

Palacino and colleagues used a proteomic approach to investigate alterations in protein expression in the ventral midbrain of 8-month-old parkin knockout mice [51]. They found 13 proteins with a decreased abundance in parkin null mice, which were mainly involved in mitochondrial respiration. In the same mice, functional assays measuring the metabolic capacity of mitochondria isolated from the striatum showed a reduced mitochondrial respiration, although the number, size and morphology of the striatal mitochondria were unchanged. In another proteomic analysis, 87 proteins, most of them involved in energy metabolism, were identified with a difference in abundance in parkin knockout mice brain compared to controls [57].

Interestingly, Stichel et al. discovered ultrastructural changes in the neurons of the $\mathrm{SN}$, the striatum and the cortex of 12 to 14-month-old parkin knockout mice in the form of cytoplasmic vacuoles and the disruption of the Golgi network and the endoplasmic reticulum (ER). In addition, a trend towards an increased number of damaged mitochondria was reported in the SN with electron dense inclusion bodies, dilated and disorganized cristae and protrusions, but this increase was not statistically significant compared to wild-type littermates [58]. However, when parkin knockout mice were crossed with transgenic mice expressing human $\alpha$-synuclein, the increase in damaged mitochondria in the $\mathrm{SN}$ was significant. Changes in the striatum or in the $\mathrm{SN}$ of young 3-month-old transgenic mice were unnoticed. The same group also measured the respiratory capacity of mitochondria isolated from the SN, striatum and cortex of aged mice. In double mutant parkin knockout/ $\alpha$-synuclein mice, a reduction of complex I activity in the SN, but not in the striatum and cortex, was found. All three brain regions were unaffected in monogenic parkin knockout mice [58].

These results indicative of a function for parkin in the regulation of mitochondrial respiration and energy metabolism were quite remarkable taking into account that parkin was generally accepted to be a cytoplasmic protein and, until recently, the link between parkin and mitochondria in mammalian cells was elusive. In contrast, Drosophila parkin (and PINK1) loss-of- 
function mutants displayed severe mitochondrial pathology, but this striking phenotype could not be replicated in mouse models. However, a key publication in 2008 by Narendra et al. convincingly showed that parkin is recruited from the cytoplasm to damaged mitochondria thereby promoting their autophagy [11]. This was followed by several reports showing that PINK1 expression is stabilized in conditions of mitochondrial impairment and essential for parkin translocation [12, 19, 59].

Thus, current insights into the cellular function of parkin and PINK1 mainly focus on their role in mitochondrial quality control. However, this has not yet been demonstrated directly in mammalian animal models. One should realize that in the cellular models, the depolarizing agent carbonylcyanide-mchlorophenyl-hydrazone (CCCP) has been used as a robust trigger of mitochondrial damage. To demonstrate a similar role of parkin and PINK1 in mammalian brain is technically much more challenging. First of all, to induce mitochondrial depolarization in vivo, systemic administration of CCCP to animal models would most probably be lethal. However, one group reported successful systemic administration of the mitochondrial uncoupler p-trifluoromethoxycarbonylcyanidephenyl-hydrazone (FCCP) in rats [60], opening an opportunity to study depolarized mitochondria in animal models. Alternatively, the depolarizing agent could be administered locoregionally in the brain or more gentle mitochondrial toxins might be required. Second, sensitive imaging techniques to detect and visualize PINK1, the migration of parkin to the mitochondria and mitophagy in vivo will probably need to be developed or refined.

\section{Cellular stress}

In addition to the changes in proteins implicated in mitochondrial respiration, alterations in proteins involved in protection from oxidative stress were discovered in the brain of parkin knockout mice [51].

Brain lysates of aged (18-20 months old) mice showed increased levels of protein carbonyls, a general marker of oxidative damage by reactive oxygen species (ROS). High levels of the lipid peroxide 4hydroxynonenal were also found in the brains of these mice. Together with a reduced antioxidant capacity in the serum, these results suggest an increased incidence of oxidative damage in the brains of aged mice [51]. On the contrary, a different study revealed no differences in the levels of protein carbonyls in the striatum and cortex of 22-month-old parkin-deficient mice [61]
Levels of reduced glutathione (GSH) were increased in the striatum of 11-month-old parkin knockout mice, but no differences were noted in oxidized glutathione (GSSG) levels [39, 50]. Moreover, elevated levels of GSH were found in neuronal cultures from ventral foetal midbrain [39]. This may suggest that parkin null mice develop a free radical defence system that protects against cell death induced by high levels of dopamine. On the other hand, a reduction of GSH levels was found in the striatum of 24-month-old parkin knockout mice, suggesting that this compensatory mechanism might be exhausted by aging [50].

In addition, a possible role for parkin in the protection against inflammation-induced neurodegeneration has been suggested [62]. Young adult (6-13 weeks old) parkin knockout and wild-type mice were repeatedly injected intraperitoneally with low-dose lipopolysaccaride (LPS) for a period of several months under various regimens. A reduction of TH-positive and NeuN-positive neurons was described in parkin knockout mice after 3 months of treatment and an additional 3 months lag time and after 6 months of administration when compared to wild-type animals. This reduction was not observed in mice treated for 3 months with LPS without the additional 3 months break. Additionally, each group of LPS-treated parkin knockout mice displayed a prolonged time to cross a narrow beam, compared to LPS-treated control mice. However, no difference in inflammatory and oxidative stress responses to the induced neuroinflammation could be detected in parkin knockout and wild-type mice. Therefore, parkin appears to influence the susceptibility of the dopaminergic neurons to inflammation-induced neurodegeneration, but the mechanism behind these observations has not been clarified, although a TNF (tumour necrosis factor) role was suggested.

\section{Susceptibility to toxin exposure}

In the absence of spontaneous dopaminergic cell loss, researchers have tried to trigger the neurodegenerative process by administration of neurotoxins in the hope that differences in susceptibility would become apparent in the parkin knockout mice.

In 2007, Thomas et al. investigated the susceptibility of the catecholaminergic neurons of the $\mathrm{SN}$ and locus coeruleus to the dopaminergic parkinsonian neurotoxin 1-methyl-4-phenyl-1,2,3,6-tetrahydropyridine (MPTP) and the noradrenergic neurotoxin N-(2-chloroethyl)-N-ethyl-2-bromobenzylamine (DSP-4) in exon 7-deleted parkin knockout mice. Neither young 
nor old parkin-deficient mice showed an increased vulnerability to acute, subacute and chronic administration of MPTP. In addition, the noradrenergic axon terminals of parkin knockout mice were not more susceptible to a single intraperitoneal injection of DSP-4 than those of wild-type animals [63]. These findings are consistent with another study in which 18-month-old exon 3-deleted parkin null mice were challenged with MPTP. There was no increase of MPTP-induced degeneration of $\mathrm{TH}-$ positive axons in the striatum of parkin-deficient mice compared to controls [42]. Additionally, METH and 6-hydroxydopamine (6-OHDA) were administered to 3-month-old exon 2-deleted parkin null mice and no increased sensitivity to these dopaminergic neurotoxins could be demonstrated [61].

On the other hand, in neuronal-enriched midbrain cultures, dopaminergic neurons of parkin knockout mice are more susceptible to rotenone-induced cell death than control mice [64]. These findings suggest that exposure to the appropriate environmental factor might be required for the development of a parkinsonian phenotype in parkin knockout mice. However, it is at present unclear why this could not yet be demonstrated in animal models.

\section{Parkin substrates}

In the past decade, several putative parkin substrates have been identified [65], but the role of these proteins in the pathogenesis of PD is not always clear. Cell division control-related protein 1 (CDCrel-1), a GTPase that accumulates in the brain of AR-JPD patients, was the first substrate to be identified [66]. Synphilin-1, a component of LBs in PD [67], was found to be ubiquitinated by parkin while the unmodified form of $\alpha$-synuclein did not interact with it [68]. However, a rare O-glycosylated $\alpha$-synuclein variant was also found to be ubiquitinated by parkin [69]. The availability of parkin knockout mice provided an ideal tool to confirm or disprove the in vivo relevance of these findings. Some groups investigated whether an accumulation of these substrates could be detected in the brains of parkin knockout and $\mathrm{qk}^{\mathrm{V}}$ mice, but the levels of CDCrel-1, synphilin-1 and $\alpha$-synuclein were similar to controls [40, 48, 70]. Furthermore, conflicting results were published about the effect of parkin deficiency on $\alpha$-synuclein mediated neurodegeneration. One study reported no effect of loss of parkin on $\alpha$-synuclein pathology in A53T $\alpha$-synuclein transgenic mice, while unexpectedly the neurodegenerative phenotype was delayed when parkin was absent in A30P a-synuclein transgenic mice $[71,72]$.

Ko and colleagues performed a systematic analysis of the levels of a set of known parkin substrates, including CDCrel-1, synphilin-1, $\alpha$-synuclein, aminoacyl-tRNA synthetase cofactor p38, Parkin associated endothelin receptor-like receptor (Pael-R), cyclin E, synaptotagmin XI and $\beta$-tubulin in the midbrain of 18-month-old exon 7-deleted parkin knockout mice [70]. Solely the levels of p38 were increased in these mice compared to wild-type age-matched littermates, in parallel with a similar increase in the brain of AR-JPD patients. Overexpression of p38 in cell culture and in the SN of mice induced cell death. These findings suggest that p38 might be an authentic parkin substrate, although the exact molecular mechanism by which p38 leads to cellular toxicity still has to be elucidated. In contrast to this study, another group could not detect an accumulation of p38 in the striatum of 12-month-old exon 3-deleted knockout mice [57].

Pael-R is a putative G-protein-coupled transmembrane protein. When overexpressed in cells, it tends to become unfolded and insoluble, which eventually leads to ER stress-induced cell death. This insoluble form of Pael-R was identified as a substrate of parkin in 2001 [73]. An interesting observation was made by Wang et al. in 2008 when they generated parkin knockout/Pael-R transgenic double mutant mice [74]. These mice exhibited a marked loss of dopaminergic and noradrenergic neurons starting at 6 months of age and further progressing until 24 months old. In the midbrain, evidence was found for ER stress and reduction in complex I activity. Furthermore, an increase in protein carbonyls was reported together with increased striatal dopamine levels in young double mutant mice. This led to the conclusion that the accumulation of Pael-R due to parkin deficiency results in chronic ER stress and excessive dopamine-mediated oxidative stress, both contributing to dopaminergic degeneration. A similar dopaminergic cell death was described in parkin knockout mice with up-regulated Pael-R expression in the $\mathrm{SN}$ achieved by adenoviral vector-mediated retrograde transduction from the striatum [41]. Although Pael-R has been found in LBs [75], its role in the pathogenesis of sporadic PD remains unclear.

Very recently, a new parkin interacting substrate, PARIS, was identified [46]. An accumulation of PARIS was found in the cingulated cortex of ARJPD patients and in the striatum and $\mathrm{SN}$ of sporadic PD patients. In the exon 7-deleted mouse line, an 
up-regulation of PARIS was reported in the striatum and ventral midbrain. Additionally, PARIS levels were increased in the ventral midbrain of conditional parkin knockout mice. PARIS is a Kruppel-associated box (KRAB) and zinc finger protein. Its accumulation represses the expression of the transcriptional coactivator PPAR $\gamma$ coactivator- $1 \alpha$ (PGC- $1 \alpha)$, leading to a down-regulation of the PGC- $1 \alpha$ dependent gene nuclear respiratory factor-1 (NRF-1). Adenoassociated viral vector-mediated overexpression of PARIS in the SN of mice led to a $40 \%$ reduction of dopaminergic neurons compared to control mice overexpressing GFP.

In conclusion, the validation of the different parkin substrates mentioned above is unclear for the moment because they lack replication across independent groups. This might be due to the variability in experimental conditions such as the mouse line, the age, etc. However, the search for accumulated parkin substrates in the brain of parkin knockout mice and in parkin-linked AR-JPD pateints was based on the initial hypothesis that loss of parkin E3-ubiquitin-ligase function impairs the ubiquitin-proteasome system (UPS) leading to a toxic accumulation of misfolded or aggregated proteins. However, recent findings have shown that activation of the UPS mediated by translocation of parkin to the mitochondria results in degradation of a wide range of mitochondrial outer membrane proteins, including mitofusin 1 and 2, Tom70, voltage dependent anion channel 1 (VDAC1), Bak, Fis1 and Tom20 [21]. This suggests that parkin has a very broad substrate range with maybe rather low specificity, at least when activated by mitochondrial dysfunction. This might explain the difficulty to prove the authenticity of the putative parkin substrates in vivo.

\section{Parkin-Q311X transgenic mouse model}

Despite not being a parkin null transgenic mouse, the bacterial artificial chromosome transgenic model expressing the truncated human mutant parkin-Q311X in dopaminergic neurons is worth to be mentioned here as a dominant-negative loss-of-function model. Its importance lies in the fact that it is the only parkin model that clearly mimics some of the key characteristics of PD. At 16 months of age but not younger, these mice exhibited a $40 \%$ reduction in $\mathrm{TH}-$ positive neurons and a $30 \%$ reduction in total neuron number in the SN compared to age-matched control littermates. In addition, a loss of striatal dopamine terminals was confirmed. Late-onset hypokinetic motor deficits were also reported as assessed by the automated open-field test, the challenging beam traversal, the cylinder test and the adhesive removal test. Furthermore, significantly reduced levels of dopamine and DOPAC were found in the striatum of 19 to 21-month-old mice. Interestingly, parkin-Q311X mice showed a progressive accumulation of proteinase $\mathrm{K}$ (PK) resistant $\alpha$-synuclein in the midbrain neurons. This PK-resistant $\alpha$-synuclein co-localized with the oxidative stress marker 3-nitrotyrosine. In summary, this model exhibits an age-dependent phenotype that displays several key features of PD and deserves further characterization [76].

\section{PINK1}

The phosphatase and tensin homolog (PTEN)induced kinase 1 (PINK1) gene was first shown to be up-regulated by the tumour suppressor gene PTEN in cancer cell lines and tissues with higher metastatic potential [77, 78]. In 2001, Valente et al. performed a genome wide homozygosity screen in a large Sicilian family with AR-JPD [79]. Linkage analysis led to the identification of a novel locus, named PARK6, spanning a $12.5 \mathrm{cM}$ region of the short arm of chromosome 1 (1p36). Subsequently, linkage to PARK6 was confirmed in several European and Asian consanguineous families [80, 81]. In 2004, PINK1 was identified as the gene responsible for the PARK6-associated parkinsonism in a Spanish family presenting a homozygous truncating mutation (W437X) and two Italian families with an homozygous missense mutation (G309D) [82]. In the following years, prevalence studies have reported that PINK1 mutations account for 1 to $9 \%$ of AR-JPD [83-87] and for 2 to $4 \%$ of sporadic $\mathrm{PD}[88,89]$ depending on the ethnic origin of the patients.

The PINK1 gene extends over eight exons $(\sim 1.8$ kilobases) and encodes a 581-amino acid protein with a predicted molecular mass of $62.8 \mathrm{kDa}$. PINK1 is ubiquitously expressed in all adult human tissues, but most abundantly in skeletal muscle, heart and testis [77]. The PINK1 mRNA distribution has been mapped throughout the rodent and human brains, revealing a uniform expression pattern of PINK1 in most brain regions, including $\mathrm{SN}$ and striatum [90, 91]. The presence of PINK1 has been reported in LBs of patients with sporadic $P D$ and $P D$ associated with heterozygous PINK1 mutations $[92,93]$. However, both publications used in-house made antibodies and therefore the specificity of the antibody and the validity of the results can- 
not be verified. Conversely, the sole neuropathological report from a PD patient associated with PARK6 mutations showed that PINK1-linked parkinsonism induces LB pathology [94]. Increased $\alpha$-synuclein aggregation was reported in PINK1-deficient cell culture models [95] but this phenomenon has not been studied in detail in rodents. PINK1 knockout mice do not clearly display $\alpha$-synuclein aggregates. Only a nonsignificant increase in $\alpha$-synuclein protein levels but in contrast, a relevant decrease in mRNA levels have been reported in the brain stem and midbrain of 24month-old PINK1 knockout mice [96]. These results suggest that $\alpha$-synuclein degradation and re-synthesis might be impaired, indicating a hurdle in protein clearance.

Since the currently available PINK1 antibodies have been shown to exhibit poor specificity and/or sensitivity, especially related to mouse PINK1, the reported distribution of PINK1 protein will need confirmation when better quality PINK1 antibodies become available. Although the lack of sensitive antibodies has hindered the reliable determination of its exact cellular location, PINK1 has been described in both the cytosolic [97-100] and the mitochondrial fractions [92, 93, 101-103]. Nevertheless, the presence of a 34-amino acid $\mathrm{N}$-terminal mitochondrial targeting motif supports its mitochondrial location, but the kinase domain may be facing the cytoplasm which allows possible interactions with cytosolic proteins [104].

The kinase catalytic domain (residues 156 to 509 ) is highly conserved and is homologous to the putative serine/threonine protein kinases of the calcium/calmodulin family. Mutations throughout the whole PINK1 gene have been described in PD patients, including missense, nonsense, point mutations, truncations and whole gene heterozygous deletions [105]. However, the bulk of mutations has been found in the kinase domain which leads to a loss of kinase activity $[82,88,97,106]$. It remains unclear whether mutations outside the kinase domain negatively affect PINK1 kinase activity. It has been reported that deletion of the C-terminal domain of PINK1 up-regulates [101] but also down-regulates [107] the kinase activity. The discrepancy in C-terminal tail function is likely caused by the use of different heterologous systems to express PINK1. More robust and reproducible experiments are required in order to assess the effect of mutations in the C-terminal region of PINK1 in patients with PD. Nonetheless, PD-linked PINK1 mutations can be considered as loss-of-function mutations that alter the kinase activity, presumably leading to mitochondrial dysfunction and instability.

\section{Generation of PINK1 loss-of-function rodents}

Up till now, seven mouse models have been developed as rodent models to study the consequences of PINK1 deficiency (Table 2). Five of these models have been generated by classical transgenesis where gene expression is disrupted by homologous recombination in embryonic stem cells and the targeted gene region is substituted by a selection cassette. Most of these studies have used a phosphoglycerate kinase (PGK) promoter-driven neomycin phosphotransferase (neo) expression cassette to disrupt normal gene expression. Via classical transgenesis, knocking out PINK1 has been accomplished by germ-line deletion of exon 1 , exons 2 to 5 , exons 4 to 5 and exons 4 to 7 [108-112].

The targeted deletion of exon 1 removed the start codon and the initial nucleotides of the coding sequence. Two mouse lines with a deletion of exon 1 were recently described [108, 109]. Our group has generated mice on a mixed genetic background where 129/Ola embryonic stem cells carrying the desired homologous recombination were injected into C57BL/6J blastocysts and chimeric offspring was further bred to a C57BL/6Jtyr ${ }^{\mathrm{c}-2 \mathrm{~J}}$ genetic background [108]. Wang et al. generated the mice on a mixed C57BL6J/129Sv genetic background [109].

Deleting the other exons resulted in the removal of the majority of the kinase domain. The deletion in mice of most of exon 2 and all of exons 3 to 5 was achieved injecting embryonic stem cells derived from the $129 \mathrm{SvEv}^{\mathrm{Brd}}$ strain and chimeric mice were bred to C57BL/6J albino mice [110]. The mouse line with deletion of exons 4 and 5 was generated in a mixed C57BL6J/129Sv genetic background [111]. As for the exons 4 to 7-deleted mouse line, C57BL6/129Sv embryonic stem cells were injected into blastocysts of C57BL/6 or BalbC mice. Chimeric mice were further intercrossed with C57BL6/129Sv hybrid mice to obtain the final homozygous mutant mice [112].

A very interesting approach performed by Gispert and colleagues consisted in the insertion of the G309DPINK1 pathogenic mutation into the orthologous mouse locus [96]. This was achieved using a neomycin selection marker, flanked by LoxP sites, engineered in intron 5. In this case, pure inbred $129 / \mathrm{SvEv}$ background mice were used to obtain homozygous mutant and wild-type mouse lines.

Alternatively, RNA interference has successfully been used to develop conditional PINK1 knockdown transgenic mice, which were generated by pronuclear 
Table 2

Key features of PINK1 knockout and knockdown mouse models

\begin{tabular}{|c|c|c|c|c|c|c|c|}
\hline Characteristics & $\begin{array}{c}\text { Kitada et al., } \\
\text { 2007; Gautier et al., } \\
2008\end{array}$ & $\begin{array}{l}\text { Zhou et al., } \\
2007\end{array}$ & $\begin{array}{c}\text { Wood-Kaczmar } \\
\text { et al. 2008; } \\
\text { Gandhi et al. } 2009\end{array}$ & $\begin{array}{c}\text { Gispert et al., } \\
2009\end{array}$ & $\begin{array}{c}\text { Heeman et al., } \\
2011^{\ddagger}\end{array}$ & $\begin{array}{l}\text { Wang et al., } \\
2011\end{array}$ & $\begin{array}{c}\text { Akundi et al., } \\
2011\end{array}$ \\
\hline \multicolumn{8}{|l|}{ Development of the model } \\
\hline Exon(s) targeted & $4-7$ & & $2-5$ & & 1 & 1 & $4-5$ \\
\hline Other technology & & shRNA & & G309D & shRNA & & \\
\hline \multicolumn{8}{|c|}{ DAergic neurons \& DA metabolism } \\
\hline Number in $\mathrm{SN}$ & $=(2-3 ; 8-9 \mathrm{~m})$ & $=(6 \mathrm{~m})$ & & $=(18 \mathrm{~m})$ & & & $=(12 \mathrm{~m})$ \\
\hline DA levels & $=(2-3 ; 8-9 \mathrm{~m})$ & $=(6 \mathrm{~m})$ & & $\downarrow(9 ; 22-24 \mathrm{~m})$ & & & $\downarrow(6 ; 8 ; 12 \mathrm{~m})$ \\
\hline Evoked DA release & $\downarrow(2-3 \mathrm{~m})$ & & & & & & \\
\hline DOPAC levels & $=(2-3 ; 8-9 \mathrm{~m})$ & $=(6 \mathrm{~m})$ & & & & & \\
\hline HVA levels & $=(2-3 ; 8-9 \mathrm{~m})$ & $=(6 \mathrm{~m})$ & & & & & \\
\hline \multicolumn{8}{|l|}{ Other neuropathology } \\
\hline Catecholamine release & $\downarrow(2-3 \mathrm{~m})$ & & & & & & \\
\hline LTP induction & $\downarrow(2-3 \mathrm{~m})$ & & & & & & \\
\hline LTD induction & $\downarrow(2-3 \mathrm{~m})$ & & & & & & \\
\hline \multicolumn{8}{|l|}{ Mitochondria } \\
\hline Morphology & $=(3-4 ; 24 \mathrm{~m})$ & & & Fragmentation & Fragmentation & Fragmentation & \\
\hline Size & $\uparrow(3-4 ; 24 \mathrm{~m})$ & & & & & & \\
\hline Number & $=(3-4 ; 24 \mathrm{~m})$ & & & & $\uparrow$ & & \\
\hline Mass & & & & $=(21 \mathrm{~m})$ & $\uparrow$ & & \\
\hline Respiration deficiency & c.I+II (3-4 m) & & & c.I+III+IV & $=$ & & \\
\hline Membrane potential & $\downarrow \ddagger$ in $[127]$ & & $\downarrow$ & $\downarrow(6 ; 12 \mathrm{~m})$ & $\downarrow$ & $\downarrow$ & \\
\hline ATP levels & $\downarrow \ddagger$ in $[127]$ & & & $\downarrow(6 ; 12 \mathrm{~m})$ & $\downarrow$ & & \\
\hline $\mathrm{Ca}^{2+}$ levels & & & $\downarrow$ & & $\downarrow$ & & $\downarrow(2 \mathrm{~m})$ \\
\hline \multicolumn{8}{|c|}{ Oxidative stress susceptibility } \\
\hline Aconitase activity & $\downarrow$ & & & & & & \\
\hline $\mathrm{H}_{2} \mathrm{O}_{2}$ exposure & $\uparrow(3-4 \mathrm{~m})$ & & & & & $\uparrow$ & \\
\hline Heat-shock treatment & $\uparrow(3-4 \mathrm{~m})$ & & & & & & \\
\hline ROS formation & $=$ & & $\uparrow$ & & & $\uparrow$ & $=$ \\
\hline \multicolumn{8}{|l|}{ Neurotoxin vulnerability } \\
\hline 6-OHDA & $=$ & & & & & & \\
\hline Rotenone & $=$ & & $\uparrow$ & & & $\uparrow$ & \\
\hline Paraquat & $=$ & & & & & & \\
\hline \multicolumn{8}{|l|}{ Response to drugs } \\
\hline D1 receptor agonist & LTP restored & & & & & & \\
\hline $\mathrm{D} 1+\mathrm{D} 2$ agonists & LTD restored & & & & & & \\
\hline Amphetamine & LTD restored & & & & & & \\
\hline L-DOPA & LTD restored & & & & & & \\
\hline \multicolumn{8}{|l|}{$\begin{array}{l}\text { L-DUPA } \\
\text { Locomotor activity }\end{array}$} \\
\hline Open field test & & & & $\downarrow(16 \mathrm{~m})$ & & & \\
\hline Rotarod & & $=$ & & & & & \\
\hline
\end{tabular}

$\uparrow:$ increase, $\downarrow$ : decrease,=: no changes (in comparison to control); shRNA: short-hairpin RNA; DA: dopamine; SN: substantia nigra; DOPAC: dihydroxyphenylacetic acid; HVA: homovanillic acid; LTP: Long-Term Potentiation; LTD: Long-Term Depression; ATP: adenosine triphosphate; $\mathrm{Ca}^{2+}$ : calcium; c: complex; $\mathrm{H}_{2} \mathrm{O}_{2}$ : hydrogen peroxide; MPTP: 1-methyl-4-phenyl-1,2,3,6tetrahydropyridine; 6-OHDA: 6-hydroxydopamine; L-DOPA: L-3,4-dihydroxyphenylalanine; $\ddagger$ : in mouse embryonic fibroblasts; mice ages are indicated in brackets if available, m: month(s). 
injection of a shRNA (short-hairpin RNA) construct under the control of a Cre-LoxP-inducible mouse U6 promoter into C57BL6/SJL fertilized eggs [113]. PINK1 transgenic knockdown mice were backcrossed to FVB genomic background for at least five generations before they were used for experiments. Similarly, our group has recently used lentiviral vectors encoding a shRNA against mouse PINK1 to generate transgenic PINK1 knockdown mice (Heeman et al. unpublished data).

Due to the lack of reliable PINK1 antibodies, all studies have proved the absence of PINK1 by means of Northern blot analysis and/or quantitative real-time RT-PCR (qPCR). Overall, the expression of PINK1 mRNA was reduced by 93 to $97 \%$ as assessed by qPCR. Only one study has not reported a solid evidence for the absence of PINK1 in their knockout transgenic offspring [110].

The information about the general appearance, lifespan, breeding behaviour and body weight of the different PINK1-deficient transgenic mouse lines generated until now is generally vaguely mentioned in literature. A single study has reported a significant loss in body weight in mice at both 10 and 16 months old [96].

\section{Neurotransmission systems and metabolism}

Synaptic function has repeatedly been studied in PINK1-deficient mice. However, contradictory results on dopamine metabolism complicate reaching a clear conclusion. On the one hand, PINK1 knockout and knockdown mice did not display significant changes in either striatal dopamine content or in its major metabolites, DOPAC and HVA, across different studies in which mice were aged 2 to 24 months [112, 113]. On the other hand, two studies have reported a reduction of approximately $25 \%$ in striatal dopamine levels in PINK1 knockout mice at 6, 9, 12 and 22 months old [96, 111]. The latter was explained in one study by an increased dopamine metabolism since dopamine turnover was found to be higher in PINK1deficient mice aged 6 to 12 months compared to age-matched wild-type littermates [111]. Intriguingly, triple parkin, PINK1 and DJ-1 knockout mice showed an increase in dopamine, DOPAC and HVA levels at 24 months old, but not at 16 months old, compared to age-matched control mice. It remains unclear how the triple knockout mice displayed an age-dependent increase of dopamine levels in striatum with normal TH levels [114]. Surprisingly, all current models failed to reproduce a meaningful degeneration of both the dopaminergic cells in the $\mathrm{SN}$ and its terminal projections to the striatum [96, 111-115].

PINK1 knockout mice presented a reduction in both evoked dopamine release in striatal slices and total catecholamine release in dissociated adrenal chromaffin cells. In addition, corticostriatal Longterm Potentiation (LTP) and Depression (LTD) was impaired, compromising the normal function of the nigrostriatal circuit and leading to an altered synaptic plasticity. LTD induction could be rescued with D1/D2 receptor agonists, amphetamine and L-3,4dihydroxyphenylalanine (L-DOPA), supporting the fact that evoked dopamine release is the mechanism underlying LTD impairments [112]. However, such deficits represent a functional rather than structural defect since they cannot be ascribed to a diminished number of dopaminergic neurons in the SN. Further electrophysiological studies in these mice showed that their striatal neurons were sensitive to group II metabotropic glutamate (mGlu) receptor activation [116]. The relevance of this finding is not yet clear since little is known about the functional link of these circuitries in monogenic forms of PD and the contribution of mGlu receptors to the modulation of the basal ganglia in general human physiology. A recent electrophysiological study has demonstrated hyperexcitability of SN dopaminergic neurons in PINK1-null mice. As a result of an impaired calcium $\left(\mathrm{Ca}^{2+}\right)$ release coming from the endoplasmatic reticulum and the mitochondria, small conductance $\mathrm{Ca}^{2+}$-activated potassium channels displayed a functional reduction in their activation, leading to these irregular firing patterns in SN [117]. The origin of this observed hyperexcitability of the dopaminergic neurons in SN might be that mitochondrial damage increases the vulnerability of PINK1-deficient mice by means of a disrupted energy balance and overt oxidative stress, as will be discussed below.

\section{Behaviour}

Since PD patients clearly display both motor and non-motor impairments, the best way to assess the disease symptoms in a new animal model is by running a battery of behavioural tests. However, an extensive behavioural study of PINK1-deficient mice has not yet been published. Indeed, only two groups have reported data about the behaviour of these mice.

Evaluation of coordination and balance by performance in the rotarod test showed no differences in PINK1 knockout and knockdown mice when compared to wild-type mice $[96,113]$. However, PINK1 
knockout mice displayed a reduced locomotor activity at 16 months old as assessed by the open field test [96]. These mice exhibited a marked decrease in the measures for total distance, horizontal activity, movement time, stereotypy count and centre distance. However, the impairment of spontaneous movement did not affect the lifespan of the animals. Strength, coordination and anxiety were also normal in these mice. Moreover, no deficit was found in non-motor functions such as sweating under pharmacological provocation or startle reflexes to acoustic stimuli. The latter provides an insight into the function of the brain stem locus coeruleus noradrenergic neurons, which control acoustic response and has been documented to be a pathological feature of AR-JPD patients [30, $33,118]$.

These two reports are in agreement with the fact that motor deficits might be triggered by damaged dopaminergic neurotransmission but are clearly not explained by an onset in dopaminergic nigrostriatal neurodegeneration. In our own PINK1 knockdown transgenic mice, we observed mild but progressive hypokinetic locomotor deficits and uncoordinated gait dynamics with aging (Heeman et al., unpublished data) although no differences in the number of nigral dopaminergic neurons or striatal dopamine and DOPAC levels were detected. It is also possible that the deficit in cellular ATP levels and the decrease of mitochondrial ATP synthesis observed in PINK1-deficient cells result in a decline in locomotion, especially when mice are challenged. This could indicate that impaired behaviour in these mice might be due to the failure of PINK1 to maintain an optimal energy balance in mitochondria.

PD patients with PINK1 gene mutations present several non-motor symptoms such as anxiety in combination with other psychiatric disorders like psychosis, obsessive behaviour, drug addiction and depression [94, 119, 120]. Behavioural tests to assess this kind of features in animal models of PD are still an issue to be able to study the molecular mechanisms behind the non-motor symptoms [121]. Despite the difficulties to determine non-motor signs in mice, symptoms such as anxiety have already being described in other animal models for PD. For instance, as mentioned previously in this review, an anxiety-like behaviour was observed in parkin knockout mice [42]. It is believed that the appearance of these symptoms precede the onset of the motor problems. It might be due to the fact that other regions are affected when the patient starts to develop the disease, long before the death of the dopaminergic neurons and the onset of the motor impairments
[3]. Consequently, non-motor symptoms are equally important during the diagnosis of AR-JPD patients, although the mechanisms leading to their manifestation remain uncertain [122].

\section{Mitochondrial morphology and function}

Since PINK1 was found to be a mitochondrial kinase, it was considered as the first protein to provide a direct link between mitochondrial dysfunction and PD pathogenesis $[88,123]$. Therefore, most of the studies of PINK1-deficient rodent models focused on the investigation of mitochondrial dynamics and the molecular mechanisms that lead to neurodegeneration of SN dopaminergic neurons in PARK6 PD patients. It is known that PINK1 modulates mitochondrial morphology and dynamics by interacting with components of the fusion/fission machinery [13]. While current data in Drosophila clearly point to a role for PINK1 in inhibiting mitochondrial fusion [103, 124], studies in mammalian cells have shown subtle and variable results. Most of the studies indicate a potential role for PINK1 in promoting mitochondrial fusion as reported in human cells and primary fibroblasts from PINK1PD patients [100, 125, 126]. These differences across species might reflect a cell type-dependent role for PINK1 in mitochondrial fusion/fission. Nevertheless, mitochondrial fusion is crucial for the maintenance of several functions in mitochondria such as respiration and membrane potential.

In general, no gross changes were noticed in mitochondrial number and ultrastructure when analyzing the striatum of PINK1 knockout mice at 3 and 24 months old [115]. This is in agreement with another report where the overall structure of mitochondria was also unaltered [96]. However, both studies have reported other morphological abnormalities in PINK1 knockout mice such as the presence of fewer fragmented mitochondria and an increase in the number of larger and aggregated mitochondria in perinuclear localization under proteasomal stress conditions [96, 115]. In addition, mouse embryonic fibroblasts derived from PINK1 knockout mice exhibited fragmented mitochondria when cultured in galactose medium [127]. We also reported a moderate increase in mitochondrial fragmentation together with a slight increase in mitochondrial mass using PINK1 knockout mouse embryonic fibroblasts, suggesting that the ablation of PINK1 induces an accumulation of depolarized and fragmented mitochondria [108]. The fragmented mitochondrial network observed in these mice supports a fusion promoting role for PINK1 in the cell. 
In terms of mitochondrial function, a reduced mitochondrial membrane potential $\left(\Delta \psi_{\mathrm{m}}\right)$ has repeatedly been reported in cells derived from PINK1 knockout mice $[96,108,109,127-129]$. The $\Delta \psi_{\mathrm{m}}$ is essential for the normal function of mitochondria and is the key regulator of its structural integrity. However, explanations for the depolarised $\Delta \psi_{\mathrm{m}}$ are contradictory across different publications. On the one hand, several studies have reported a normal activity of the respiratory chain complexes I to IV of the oxidative phosphorylation system using different PINK1 knockout and knockdown cell lines [108, 125, 129]. On the other hand, mitochondrial respiration was impaired for complexes I and II in striatum but not in cortex of 3-month-old PINK1 knockout mice, which implies a specific functional association to the dopamine metabolism [115]. Still, other studies in PINK1 knockout mice have reported respiration deficiencies in complex I [128] and complexes I, III and IV [96]. Evidence for unchanged levels of protein respiratory chain complexes in all these studies suggests that the respiratory deficiency is functional rather than structural. We cannot discard the hypothesis that respiratory chain complexes might work in a less efficient way in studies where no clear deficit in their activity was detected. Reports on the kinetics of isolated PINK1-deficient mitochondria strengthened the idea that the source of the $\Delta \psi_{\mathrm{m}}$ depolarisation is a defect in the substrate oxidation of the respiratory chain [127, 129]. For this reason, the conflicting data do not facilitate the understanding of the mechanism that leads to the depolarized mitochondrial membrane potential, although the recently identified role of PINK1 in clearance of dysfunctional mitochondria, as mentioned previously, might explain most observations. However, further investigation into the precise molecular link must be carried out.

Several studies have shown that PINK1 regulates mitochondrial calcium dynamics and plays a critical role in energy balance. We found that its depletion leads to an impairment in $\mathrm{Ca}^{2+}$ entry into the mitochondria and a significant reduction of the cytosolic $\mathrm{Ca}^{2+}$ levels in conditions of high energy demand [108]. In addition, another study has reported that mitochondria of PINK1-deficient neurons accumulate higher basal levels of $\mathrm{Ca}^{2+}$ in the matrix and display a reduced mitochondrial $\mathrm{Ca}^{2+}$ storage capacity associated with $\mathrm{Ca}^{2+}$ overload [129]. Similarly, mitochondria from brains of 2-month-old PINK1 knockout mice showed a lower $\mathrm{Ca}^{2+}$ load capacity indicated by the earlier opening of the mitochondrial permeability transition pore [111]. Concomitant to changes in $\mathrm{Ca}^{2+}$ homeostasis, we observed a decrease in mitochondrial ATP synthesis under conditions of high energy demand in PINK1depleted cells [108]. Similar changes were observed for some PINK1 mutants, suggesting a dominant negative effect [95]. Moreover, cellular ATP levels were reported to be reduced in the absence of PINK1 [96, 127].

In conclusion, both morphological and functional anomalies were reported in PINK1-deficient mitochondria pointing to impairments in mitochondrial bioenergetics, despite some inconsistencies in the exact phenotypic features between different studies. However, the exact molecular cause of the general mitochondrial dysfunction in PINK1 knockout mouse models still needs to be elucidated.

\section{Cellular stress}

The neuroprotective effect of PINK1 has been demonstrated in several cell culture models, where overexpression or suppression of PINK1 was combined with different forms of cellular stress. The initial study of Valente et al. reported that wild-type PINK1, but not its PD-associated mutants, protected against cell death induced by proteasome inhibition [82], which was subsequently confirmed by other groups [130-132]. Down-regulation of PINK1 or overexpression of PARK6 disease-causing mutations appeared to reduce basal cell viability [110, 123, 133-135]. In addition, PINK1 has been shown to confer protection against hydroxyl radicals $\left(\mathrm{H}_{2} \mathrm{O}_{2}\right)[136,137]$.

Similarly, isolated cortical mitochondria from PINK1 knockout mice were more sensitive to exposure to environmental cellular stress inducers like $\mathrm{H}_{2} \mathrm{O}_{2}$ and mild heat shock, despite normal antioxidant enzyme levels [115]. It has been reported that cultured dopaminergic neurons from PINK1 knockout mice exhibited enhanced ROS formation that could be rescued by introducing wild-type PINK1 [109, 129]. Taken together, these findings suggest that oxidative stress caused by environmental insults might exacerbate PINK1-related mitochondrial dysfunction.

Recently, Akundi et al. showed that phosphorylated c-Jun accumulates in the SN of PINK1 knockout mice [111]. c-Jun is the main substrate of the proapoptotic Jun N-terminal kinase (JNK), a member of the MAP (mitogen-activated protein) kinase family implicated in neuronal cell death [138]. In addition, the lack of PINK1 resulted in decreased cytokineinduced nuclear factor kappa- $\beta$ (NF-кB) activity in mouse embryonic fibroblasts and increased levels of inflammatory cytokines (IL-1b, IL-10 and IL12) in the striatum of PINK1 knockout mice after 
peripheral LPS treatment. Moreover, striatal gene expression profiling of 2-month-old knockout and control mice revealed that PINK1-deficiency activates genes linked to anti-inflammatory response and stress-inducible transcription factors, whereas genes associated with pro-inflammatory and apoptotic responses were down-regulated. These novel findings indicate that the ablation of PINK1 causes altered expression of genes that regulate innate immune responses and predispose neurons to inflammation and oxidative stress-induced apoptosis. How the increased JNK signalling and immune response pathway are linked to the increased mitochondrial $\mathrm{Ca}^{2+}$ sensitivity reported in the same study is at present unclear. The authors speculate that they might all contribute to the observed increase in dopamine turnover and lead to a higher vulnerability of the nigrostriatal system to degenerate.

\section{Susceptibility to toxin exposure}

Since no spontaneous overt cell loss is observed in the PINK1 knockout rodent models, another valid option is to consider a 'dual hit' strategy, whereby the genetic loss-of-function animals are combined with environmental challenges such as dopaminergic neurotoxins. At the same time, the use of neurotoxins, which are known to interfere with respiratory function and to induce ROS production, provides a suitable way to evaluate the sensitivity of mitochondria.

The effect of a PD-linked toxin, rotenone, has been studied in PINK1-deficient cells. Indeed, cultured dopaminergic neurons from PINK1 knockout mice appeared to be more sensitive to rotenone-induced cell death [109, 129]. In 2008, Haque et al. reported that knockdown of PINK1 significantly increased the sensitivity of primary cortical neurons to the effects of the MPTP neurotoxin [99]. Conversely, the presence of PINK1 proved to protect primary cortical neurons against the same induced toxicity. In vivo, experiments targeting the $\mathrm{SN}$ of adult mice by adenoviral vector-mediated retrograde transport from the striatum showed that overexpression of wild-type PINK1 provided protection against MPTP treatment. Moreover, these experiments revealed that PINK1-mediated neuroprotection needs the presence of the kinase domain since its mutant forms failed to provide any protection after MPTP administration, both in cell culture and in vivo [99].

It was generally believed that mitochondrial localization of PINK1 is indispensable to exert its neuroprotective function. However, Haque et al. showed that N-terminal truncated PINK1 still provided protection against MPTP toxicity, both in cell culture and in vivo [99]. Therefore, it remains to be clarified whether the mitochondrial targeting motif is essential for the neuroprotective role of PINK1, although it has been shown that mitochondrial localization of PINK1 can also occur independently of the mitochondrial targeting motif [100].

Finally, PINK1 is undoubtedly important for the survival of neurons under oxidative stress since its depletion increases the sensitivity to diverse neurotoxic insults while its overexpression exerts a neuroprotective effect. However, here again, the exact molecular mechanism remains to be demonstrated, although the recently identified role of PINK1 in mitophagy and mitochondrial integrity may provide a satisfactory explanation.

\section{CONCLUSION}

We have here reviewed all currently available knockout rodent models for parkin and PINK1 which are the most frequent causative genes of recessively inherited early-onset PD. Both models have contributed to the understanding of the molecular mechanisms involved in the pathogenesis of the disease. Indeed, they convey the idea that mitochondrial impairment and oxidative stress are involved in PD pathology. However, they all present a negligible neurodegenerative pattern in the substantia nigra pars compacta, a region clearly affected in PD patients. Moreover, the results obtained with these models differed greatly across studies, implying that the specific procedure to generate the animal model influences the outcome. For example, it is believed that behavioural deficits in rodents are dependent on the background of the strain $[56,139]$. Hence, the background mouse strain used to generate the parkin and PINK1 loss-of-function models may lead to significant differences in the results of the experiments.

Mutations in several disease-causing genes have been reported until now in familial PD. However, it is still unclear whether all the proteins involved interact with each other or if they converge in a common pathway. Findings that parkin acts downstream of PINK1 in both Drosophila and mammalian neuronal cultures support the latter hypothesis $[125,140$, 141]. This implies that both proteins are involved in the same pathophysiological pathway that regulates mitochondrial stability and cell survival [12, 19, 59, 142]. However, these findings still have to be demonstrated 
in the mammalian models in vivo, which is technically much more challenging. Mitochondrial dysfunction in dopaminergic neurons has been proposed as a primary mechanism in neurodegeneration. This would lead to the accumulation of ROS, sensitivity to oxidative stress and final cell death. Intriguingly, even triple parkin, PINK1 and DJ-1 knockout mice do not show any dopaminergic cell loss [114]. Therefore, further studies are needed to fully understand the mechanistic events at the origin of the disease.

It remains to be resolved why patients harbouring parkin and PINK1 mutations develop early-onset $\mathrm{PD}$, whereas total loss of these proteins in mice fails to reproduce neurodegeneration. The fact that in other species, such as Drosophila, loss of parkin or PINK1 induces mild neurodegeneration in contrast to rodent models is also a question that needs to be addressed. The development of animal models for neurodegenerative disorders is not trivial since it requires recapitulating symptoms of a human disease in the animal [143, 144]. For instance, all rodent models developed so far for parkin and PINK1 null mutations are exclusively mouse models. However, the short lifespan of mice impedes true long-term studies to gain further knowledge about the disease progression. Another important issue that might explain the lack of clear PD features in parkin and PINK1 transgenic knockout mice is the possibility of compensatory mechanisms ongoing during embryonic development $[116,128]$. These mechanisms might not be present in Drosophila, explaining the observed mitochondrial phenotype leading to apoptotic cell death and muscle degeneration [141, 145]. In addition, it is reported that similar compensatory mechanisms occur in humans in the presymptomatic phase of the disease [146]. The premature failure of these compensatory mechanisms might contribute to the development of early-onset PD.

We propose that these compensatory issues may be overcome in rodents by viral vector-mediated transfer of the disease-causing gene or of an inhibitory shRNA sequence in adult brain. This 'local transgenesis' is a highly flexible method which can be applied in different mammalian species, broadening the generation of models up to more human-like species such as primates. In addition, the parallel comparison with control viral vectors allows to rule out aspecific affects due to protein overexpression. Finally, specific targeting of the brain region of interest, in this case the dopaminergic cells of the SN makes it feasible to evaluate cell type-specific differences in susceptibility [147, 148]. In fact, it has already been proven that lentivi- ral and adeno-associated viral vectors overexpressing $\alpha$-synuclein induce PD-like neuropathology and neurodegeneration in rodents and primates, whereas the transgenic models fail to display the latter crucial feature [149-153]. In the case of parkin and PINK1, where the protein loss of function triggers the disease, stable knockdown of gene expression can be achieved by viral vector-mediated RNA interference (RNAi) after stereotactic injection in adult SN [154, 155]. Adult knockdown achieved by combining RNAi with viral vector technology has not yet been reported for parkin or PINK1. However, adult local knockout was recently achieved by a similar elegant approach: Cre-expressing lentiviral vectors were injected in the $\mathrm{SN}$ of adult parkin $^{\mathrm{Flx} / \mathrm{Flx}}$ mice resulting in a loss of parkin protein and a progressive loss of the dopaminergic neurons [46]. Although this approach has not yet been independently verified, the generation of genetic models of PD by means of viral vectors constitutes an alternative approach to classical transgenesis and a promising tool for animal modelling of PD [156]. Evidently, the misinterpretation of phenomena due to possible toxic effects of viral vectors should be avoided by including the proper controls.

Another less explored option that might mimic more closely the physiological condition of the parkin and PINK1 mutation carriers is the generation of knock-in mice in which the normal copy of the gene is replaced by a mutant form of the gene. It has been reported that mutations not always lead to a total obliteration of the protein activity [157]. Therefore, the advantage of knocking in the mutant gene is that the compensatory mechanisms that can arise by completely knocking out the gene and abolishing the protein function are circumvented.

A final possible explanation for the failure to obtain a PD phenotype in the present knockout models is, as a consequence of the well-controlled housing of all laboratory animals, the lack of exposure to the appropriate environmental triggers. Attempts to study the influence of the environment were made by administering neurotoxins to the mouse models. The fact that PINK1 mutants and PINK1 knockdown animals are more vulnerable to different neurotoxins than control animals might argue in favour of a role of the environment. However, in the parkin knockout models, data are much less convincing. Also, neurotoxins probably poorly mimic environmental influences. An important counterargument is the fact that all humans with two mutant PINK1 and parkin alleles develop the disease, irrespective of their environment [25, $158]$. 
Finally, despite the apparent current shortcomings as parkinsonian models, both parkin and PINK1 transgenic knockout mice are still valuable by offering a basic insight into the different pathways and proteins involved in the aetiology of PD. As models, parkin and PINK1-deficient rodents still hold some questions and challenges that are essential to tackle soon. We believe that other approaches, such as gene silencing mediated by viral vector technology, may greatly contribute to improve these models in order to further test and identify novel treatments for PD patients.

\section{ACKNOWLEDGMENTS}

This work was supported by the European FP7 ITN NEUROMODEL (PITN-GA-2008-215618), the FP7 RTD MEFOPA (HEALTH-2009-241791), the Queen Elisabeth Medical Foundation, the Institute for the Promotion of Innovation through Science and Technology in Flanders (IWT/SBO 80020) and the Flemish Research Foundation FWO-Vlaanderen (project G.0406.06, doctoral fellowships to A-S.V.R. and B.H.).

\section{REFERENCES}

[1] Farrer MJ (2006) Genetics of Parkinson disease: Paradigm shifts and future prospects. Nat Rev Genet, 7(4), p. 306-318

[2] Dorsey ER, et al. (2007) Projected number of people with Parkinson disease in the most populous nations: 2005 through 2030. Neurology, 68(5), 384-386.

[3] Braak H, et al. (2003) Staging of brain pathology related to sporadic Parkinson's disease. Neurobiol Aging, 24(2), $197-$ 211.

[4] Braak H, Rub U, \& Del Tredici K (2006) Cognitive decline correlates with neuropathological stage in Parkinson's disease. J Neurol Sci, 248(1-2), 255-258.

[5] Polymeropoulos MH, et al. (1997) Mutation in the alphasynuclein gene identified in families with Parkinson's disease. Science, 276(5321), 2045-2047.

[6] Spillantini MG, et al. (1997) Alpha-synuclein in Lewy bodies. Nature, 388(6645), 839-840.

[7] Satake W, et al. (2009) Genome-wide association study identifies common variants at four loci as genetic risk factors for Parkinson's disease. Nat Genet, 41(12), 1303-1307.

[8] Simon-Sanchez J, et al. (2009) Genome-wide association study reveals genetic risk underlying Parkinson's disease. Nat Genet, 41(12), 1308-1312.

[9] Shimura H, et al. (2000) Familial Parkinson disease gene product: Parkin, is a ubiquitin-protein ligase. Nat Genet, 25(3), 302-305

[10] Imai Y, Soda M, \& Takahashi R (2000) Parkin suppresses unfolded protein stress-induced cell death through its E3 ubiquitin-protein ligase activity. J Biol Chem, 275(46), 35661-35664.

[11] Narendra D, et al. (2008) Parkin is recruited selectively to impaired mitochondria and promotes their autophagy. J Cell Biol, 183(5), 795-803.
[12] Narendra DP, et al. (2010) PINK1 is selectively stabilized on impaired mitochondria to activate Parkin. PLoS Biol, 8(1), e1000298.

[13] Thomas KJ, \& Cookson MR (2009) The role of PTENinduced kinase 1 in mitochondrial dysfunction and dynamics. Int J Biochem Cell Biol, 41(10), 2025-2035.

[14] Youle RJ, \& Narendra DP (2011) Mechanisms of mitophagy. Nat Rev Mol Cell Biol, 12(1), 9-14.

[15] Springer W, \& Kahle PJ (2011) Regulation of PINK1Parkin-mediated mitophagy. Autophagy, 7(3), 266-278.

[16] Vives-Bauza C, \& Przedborski S (2011) Mitophagy: The latest problem for Parkinson's disease. Trends Mol Med, 17(3), 158-165.

[17] Deas E, Wood NW, \& Plun-Favreau H (2011) Mitophagy and Parkinson's disease: The PINK1-parkin link. Biochim Biophys Acta, 1813(4), 623-633.

[18] Geisler S, et al. (2010) The PINK1/Parkin-mediated mitophagy is compromised by PD-associated mutations. Autophagy, 6(7), 871-878.

[19] Geisler S, et al. (2010) PINK1/Parkin-mediated mitophagy is dependent on VDAC1 and p62/SQSTM1. Nat Cell Biol, 12(2), 119-131.

[20] Gegg ME, et al. (2010) Mitofusin 1 and mitofusin 2 are ubiquitinated in a PINK1/parkin-dependent manner upon induction of mitophagy. Hum Mol Genet, 19(24), 48614870 .

[21] Chan NC, et al. (2011) Broad activation of the ubiquitinproteasome system by Parkin is critical for mitophagy. Hum Mol Genet, 20(9), 1726-1737.

[22] Matsumine H, et al. (1997) Localization of a gene for an autosomal recessive form of juvenile Parkinsonism to chromosome 6q25.2-27. Am J Hum Genet, 60(3), 588-596.

[23] Kitada T, et al. (1998) Mutations in the parkin gene cause autosomal recessive juvenile parkinsonism. Nature, 392(6676), 605-608.

[24] Hristova VA, et al. (2009) Identification of a novel Zn2+-binding domain in the autosomal recessive juvenile Parkinson-related E3 ligase parkin. J Biol Chem, 284(22), 14978-14986.

[25] Lucking CB, et al. (2000) Association between early-onset Parkinson's disease and mutations in the parkin gene. NEngl J Med, 342(21), 1560-1567.

[26] Periquet M, et al. (2003) Parkin mutations are frequent in patients with isolated early-onset parkinsonism. Brain, 126(Pt 6), 1271-1278.

[27] Mata IF, Lockhart PJ, \& Farrer MJ (2004) Parkin genetics: One model for Parkinson's disease. Hum Mol Genet, $13 \mathrm{Spec}$ No 1, p. R127-R133.

[28] Ishikawa A, \& Takahashi H (1998) Clinical and neuropathological aspects of autosomal recessive juvenile parkinsonism. J Neurol, 245(11 Suppl 3), P4-P9.

[29] Mori H, et al. (1998) Pathologic and biochemical studies of juvenile parkinsonism linked to chromosome 6q. Neurology, 51(3), 890-892.

[30] Hayashi S, et al. (2000) An autopsy case of autosomalrecessive juvenile parkinsonism with a homozygous exon 4 deletion in the parkin gene. Mov Disord, 15(5), 884-888.

[31] van de Warrenburg BP, et al. (2001) Clinical and pathologic abnormalities in a family with parkinsonism and parkin gene mutations. Neurology, 56(4), 555-557.

[32] Sasaki S, et al. (2004) Parkin-positive autosomal recessive juvenile Parkinsonism with alpha-synuclein-positive inclusions. Neurology, 63(4), 678-682.

[33] Yamamura Y, et al. (2000) Autosomal recessive early-onset parkinsonism with diurnal fluctuation: Clinicopathologic 
characteristics and molecular genetic identification. Brain Dev, 22(Suppl 1), p. S87-S91.

[34] Farrer M, et al. (2001) Lewy bodies and parkinsonism in families with parkin mutations. Ann Neurol, 50(3), 293-300.

[35] Pramstaller PP, et al. (2005) Lewy body Parkinson's disease in a large pedigree with 77 Parkin mutation carriers. Ann Neurol, 58(3), 411-422.

[36] Sasaki S, et al. (2008) Involvement of spinal motor neurons in parkin-positive autosomal recessive juvenile parkinsonism. Neuropathology, 28(1), 74-80.

[37] Perez FA, \& Palmiter RD (2005) Parkin-deficient mice are not a robust model of parkinsonism. Proc Natl Acad Sci U S A, 102(6), 2174-2179.

[38] Sato S, et al. (2006) Decline of striatal dopamine release in parkin-deficient mice shown by ex vivo autoradiography. $J$ Neurosci Res, 84(6), 1350-1357.

[39] Itier JM, et al. (2003) Parkin gene inactivation alters behaviour and dopamine neurotransmission in the mouse. Hum Mol Genet, 12(18), 2277-2291.

[40] Goldberg MS, et al. (2003) Parkin-deficient mice exhibit nigrostriatal deficits but not loss of dopaminergic neurons. J Biol Chem, 278(44), 43628-43635.

[41] Kitao Y, et al. (2007) Pael receptor induces death of dopaminergic neurons in the substantia nigra via endoplasmic reticulum stress and dopamine toxicity: Which is enhanced under condition of parkin inactivation. Hum Mol Genet, 16(1), 50-60.

[42] Zhu XR, et al. (2007) Non-motor behavioural impairments in parkin-deficient mice. Eur J Neurosci, 26(7), 1902-1911.

[43] Kitada T, et al. (2000) Progress in the clinical and molecular genetics of familial parkinsonism. Neurogenetics, 2(4), 207218.

[44] West A, et al. (2002) Complex relationship between Parkin mutations and Parkinson disease. Am J Med Genet, 114(5), 584-591.

[45] Von Coelln R, et al. (2004) Loss of locus coeruleus neurons and reduced startle in parkin null mice. Proc Natl Acad Sci U S A, 101(29), 10744-10749.

[46] Shin JH, et al. (2011) PARIS (ZNF746) repression of PGC-1alpha contributes to neurodegeneration in Parkinson's disease. Cell, 144(5), 689-702.

[47] Sidman RL, Dickie MM, \& Appel SH (1964) Mutant Mice (Quaking and Jimpy) with Deficient Myelination in the Central Nervous System. Science, 144, 309-311.

[48] Lorenzetti D, et al. (2004) The neurological mutant quaking(viable) is Parkin deficient. Mamm Genome, 15(3), 210-217.

[49] Lockhart PJ, O'Farrell CA, \& Farrer MJ (2004) It's a double knock-out! The quaking mouse is a spontaneous deletion of parkin and parkin co-regulated gene (PACRG). Mov Disord, 19(1), 101-104.

[50] Rodriguez-Navarro JA, et al. (2007) Mortality: Oxidative stress and tau accumulation during ageing in parkin null mice. J Neurochem, 103(1), 98-114.

[51] Palacino JJ, et al. (2004) Mitochondrial dysfunction and oxidative damage in parkin-deficient mice. $J$ Biol Chem, 279(18), p. 18614-18622. Epub 2004 Feb 24.

[52] Oyama G, et al. (2010) Impaired in vivo dopamine release in parkin knockout mice. Brain Res, 1352, 214-222.

[53] Kitada T, et al. (2009) Impaired dopamine release and synaptic plasticity in the striatum of parkin-/- mice. J Neurochem, 110(2), 613-621.

[54] Nikulina EM, et al. (1995) Dopaminergic brain system in the quaking mutant mouse. Pharmacol Biochem Behav, 50(3), 333-337.
[55] Aarsland D, et al. (1999) Range of neuropsychiatric disturbances in patients with Parkinson's disease. J Neurol Neurosurg Psychiatry, 67(4), 492-496.

[56] Paylor R, \& Crawley JN (1997) Inbred strain differences in prepulse inhibition of the mouse startle response. Psychopharmacology (Berl), 132(2), 169-180.

[57] Periquet M, et al. (2005) Proteomic analysis of parkin knockout mice: Alterations in energy metabolism, protein handling and synaptic function. J Neurochem, 95(5), 12591276.

[58] Stichel CC, et al. (2007) Mono- and double-mutant mouse models of Parkinson's disease display severe mitochondrial damage. Hum Mol Genet, 16(20), 2377-2393.

[59] Vives-Bauza C, et al. (2010) PINK1-dependent recruitment of Parkin to mitochondria in mitophagy. Proc Natl Acad Sci U S A, 107(1), 378-383.

[60] Pandya JD, Pauly JR, \& Sullivan PG (2009) The optimal dosage and window of opportunity to maintain mitochondrial homeostasis following traumatic brain injury using the uncoupler FCCP. Exp Neurol, 218(2), 381-389.

[61] Perez FA, Curtis WR, \& Palmiter RD (2005) Parkindeficient mice are not more sensitive to 6-hydroxydopamine or methamphetamine neurotoxicity. BMC Neurosci, 6, p. 71.

[62] Frank-Cannon TC, et al. (2008) Parkin deficiency increases vulnerability to inflammation-related nigral degeneration. $J$ Neurosci, 28(43), 10825-10834.

[63] Thomas B, et al. (2007) MPTP and DSP-4 susceptibility of substantia nigra and locus coeruleus catecholaminergic neurons in mice is independent of parkin activity. Neurobiol Dis, 26(2), 312-322.

[64] Casarejos MJ, et al. (2006) Susceptibility to rotenone is increased in neurons from parkin null mice and is reduced by minocycline. J Neurochem, 97(4), 934-946.

[65] von Coelln R, Dawson VL, \& Dawson TM (2004) Parkinassociated Parkinson's disease. Cell Tissue Res, 318(1), 175184.

[66] Choi P, et al. (2003) SEPT5_v2 is a parkin-binding protein Brain Res Mol Brain Res, 117(2), 179-189.

[67] Wakabayashi K, et al. (2000) Synphilin-1 is present in Lewy bodies in Parkinson's disease. Ann Neurol, 47(4), 521523.

[68] Chung KK, et al. (2001) Parkin ubiquitinates the alphasynuclein-interacting protein, synphilin-1: Implications for Lewy-body formation in Parkinson disease. Nat Med, 7(10), 1144-1150.

[69] Shimura H, et al. (2001) Ubiquitination of a new form of alpha-synuclein by parkin from human brain: Implications for Parkinson's disease. Science, 293(5528), 263-269.

[70] Ko HS, et al. (2005) Accumulation of the authentic parkin substrate aminoacyl-tRNA synthetase cofactor, p38/JTV-1, leads to catecholaminergic cell death. J Neurosci, 25(35), 7968-7978.

[71] Fournier M, et al. (2009) Parkin deficiency delays motor decline and disease manifestation in a mouse model of synucleinopathy. PLoS One, 4(8), e6629.

[72] von Coelln R, et al. (2006) Inclusion body formation and neurodegeneration are parkin independent in a mouse model of alpha-synucleinopathy. J Neurosci, 26(14), 3685-3696.

[73] Imai Y, et al. (2001) An unfolded putative transmembrane polypeptide: Which can lead to endoplasmic reticulum stress, is a substrate of Parkin. Cell, $\mathbf{1 0 5}(7), 891-902$.

[74] Wang HQ, et al. (2008) Pael-R transgenic mice crossed with parkin deficient mice displayed progressive and selective catecholaminergic neuronal loss. J Neurochem, 107(1), 171185 . 
[75] Murakami T, et al. (2004) Pael-R is accumulated in Lewy bodies of Parkinson's disease. Ann Neurol, 55(3), 439442.

[76] Lu XH, et al. (2009) Bacterial artificial chromosome transgenic mice expressing a truncated mutant parkin exhibit age-dependent hypokinetic motor deficits, dopaminergic neuron degeneration, and accumulation of proteinase $\mathrm{K}$ resistant alpha-synuclein. J Neurosci, 29(7), 1962-1976.

[77] Unoki M \& Nakamura Y (2001) Growth-suppressive effects of BPOZ and EGR2: Two genes involved in the PTEN signaling pathway. Oncogene, 20(33), 4457-4465.

[78] Nakajima A, et al. (2003) BRPK: A novel protein kinase showing increased expression in mouse cancer cell lines with higher metastatic potential. Cancer Lett, 201(2), 195201.

[79] Valente EM, et al. (2001) Localization of a novel locus for autosomal recessive early-onset parkinsonism: PARK6, on human chromosome 1p35-p36. Am J Hum Genet, 68(4), 895-900.

[80] Valente EM, et al. (2002) PARK6-linked parkinsonism occurs in several European families. Ann Neurol, 51(1), 14-18.

[81] Hatano Y, et al. (2004) PARK6-linked autosomal recessive early-onset parkinsonism in Asian populations. Neurology, 63(8), 1482-1485.

[82] Valente EM, et al. (2004) Hereditary early-onset Parkinson's disease caused by mutations in PINK1. Science, 304(5674), 1158-1160.

[83] Healy DG, et al. (2004) PINK1 (PARK6) associated Parkinson disease in Ireland. Neurology, 63(8), 1486-1488.

[84] Rogaeva E, et al. (2004) Analysis of the PINK1 gene in a large cohort of cases with Parkinson disease. Arch Neurol, 61(12), 1898-1904.

[85] Schlitter AM, et al. (2005) Exclusion of PINK1 as candidate gene for the late-onset form of Parkinson's disease in two European populations. J Negat Results Biomed, 4, 10.

[86] Ibanez P, et al. (2006) Mutational analysis of the PINK1 gene in early-onset parkinsonism in Europe and North Africa. Brain, 129(Pt 3), 686-694.

[87] Guo JF, et al. (2010) Mutation analysis of Parkin, PINK1 and DJ-1 genes in Chinese patients with sporadic early onset parkinsonism. J Neurol, 257(7), 1170-1175.

[88] Valente EM, et al. (2004) PINK1 mutations are associated with sporadic early-onset parkinsonism. Ann Neurol, 56(3), 336-341.

[89] Tan EK, et al. (2006) PINK1 mutations in sporadic earlyonset Parkinson's disease. Mov Disord, 21(6), 789-793.

[90] Taymans JM, Van den Haute C, \& Baekelandt V (2006) Distribution of PINK1 and LRRK2 in rat and mouse brain. J Neurochem, 98(3), 951-961.

[91] Blackinton JG, et al. (2007) Expression of PINK1 mRNA in human and rodent brain and in Parkinson's disease. Brain Res, 1184, 10-16.

[92] Gandhi S, et al. (2006) PINK1 protein in normal human brain and Parkinson's disease. Brain, 129(Pt 7), 1720-1731.

[93] Murakami T, et al. (2007) PINK1: A gene product of PARK6, accumulates in alpha-synucleinopathy brains. $J$ Neurol Neurosurg Psychiatry, 78(6), 653-654.

[94] Samaranch L et al PINK1-linked parkinsonism is associated with Lewy body pathology. Brain, 133(Pt 4), p. 11281142

[95] Liu W, et al. (2009) PINK1 defect causes mitochondrial dysfunction, proteasomal deficit and alpha-synuclein aggregation in cell culture models of Parkinson's disease. PLoS One, 4(2), e4597.
[96] Gispert S, et al. (2009) Parkinson phenotype in aged PINK1deficient mice is accompanied by progressive mitochondrial dysfunction in absence of neurodegeneration. PLoS One, 4(6), e5777.

[97] Hatano Y, et al. (2004) Novel PINK1 mutations in earlyonset parkinsonism. Ann Neurol, 56(3), 424-427.

[98] Beilina A, et al. (2005) Mutations in PTEN-induced putative kinase 1 associated with recessive parkinsonism have differential effects on protein stability. Proc Natl Acad Sci U S A, 102(16), 5703-5708.

[99] Haque ME, et al. (2008) Cytoplasmic Pink1 activity protects neurons from dopaminergic neurotoxin MPTP. Proc Natl Acad Sci U S A, 105(5), 1716-1721.

[100] Weihofen A, et al. (2009) Pink1 forms a multiprotein complex with Miro and Milton, linking Pink1 function to mitochondrial trafficking. Biochemistry, 48(9), 2045-2052.

[101] Silvestri L, et al. (2005) Mitochondrial import and enzymatic activity of PINK1 mutants associated to recessive parkinsonism. Hum Mol Genet, 14(22), 3477-3492.

[102] Muqit MM, et al. (2006) Altered cleavage and localization of PINK1 to aggresomes in the presence of proteasomal stress. J Neurochem, 98(1), 156-169.

[103] Yang Y, et al. (2008) Pink1 regulates mitochondrial dynamics through interaction with the fission/fusion machinery. Proc Natl Acad Sci U S A, 105(19), 7070-7075.

[104] Zhou C, et al. (2008) The kinase domain of mitochondrial PINK1 faces the cytoplasm. Proc Natl Acad Sci U S A, 105(33), 12022-12027.

[105] Marongiu R, et al. (2008) PINK1 heterozygous rare variants: Prevalence, significance and phenotypic spectrum. Hum Mutat, 29(4), 565.

[106] Rohe CF, et al. (2004) Homozygous PINK1 C-terminus mutation causing early-onset parkinsonism. Ann Neurol, 56(3), 427-431.

[107] Sim CH, et al. (2006) C-terminal truncation and Parkinson's disease-associated mutations down-regulate the protein serine/threonine kinase activity of PTEN-induced kinase-1. Hum Mol Genet, 15(21), 3251-3262.

[108] Heeman B, et al. (2011) Depletion of PINK1 affects mitochondrial metabolism, calcium homeostasis and energy maintenance. J Cell Sci, 124(Pt 7), 1115-1125.

[109] Wang HL, et al. (2011) PARK6 PINK1 mutants are defective in maintaining mitochondrial membrane potential and inhibiting ROS formation of substantia nigra dopaminergic neurons. Biochim Biophys Acta, 12(6), 674-684.

[110] Wood-Kaczmar A, et al. (2008) PINK1 is necessary for long term survival and mitochondrial function in human dopaminergic neurons. PLoS One, 3(6), e2455.

[111] Akundi RS, et al. (2011) Increased mitochondrial calcium sensitivity and abnormal expression of innate immunity genes precede dopaminergic defects in Pink1-deficient mice. PLoS One, 6(1), e16038.

[112] Kitada T, et al. (2007) Impaired dopamine release and synaptic plasticity in the striatum of PINK1-deficient mice. Proc Natl Acad Sci U S A, 104(27), 11441-11446.

[113] Zhou H, et al. (2007) Silencing of the Pink1 gene expression by conditional RNAi does not induce dopaminergic neuron death in mice. Int J Biol Sci, 3(4), 242-250.

[114] Kitada T, et al. (2009) Absence of nigral degeneration in aged parkin/DJ-1/PINK1 triple knockout mice. $\mathrm{J} \mathrm{Neu-}$ rochem, 111(3), 696-702.

[115] Gautier CA, Kitada T \& Shen J (2008) Loss of PINK1 causes mitochondrial functional defects and increased sensitivity to oxidative stress. Proc Natl Acad Sci U S A, 105(32), 1136411369. 
[116] Martella G, et al. (2009) Enhanced sensitivity to group II mGlu receptor activation at corticostriatal synapses in mice lacking the familial parkinsonism-linked genes PINK1 or Parkin. Exp Neurol, 215(2), 388-396.

[117] Bishop MW, et al. (2010) Hyperexcitable substantia nigra dopamine neurons in PINK1- and HtrA2/Omi-deficient mice. J Neurophysiol, 104(6), 3009-3020.

[118] Gouider-Khouja N, et al. (2003) Autosomal recessive parkinsonism linked to parkin gene in a Tunisian family. Clinical: Genetic and pathological study. Parkinsonism Relat Disord, 9(5), 247-251.

[119] Bonifati V, et al. (2005) Early-onset parkinsonism associated with PINK1 mutations: Frequency, genotypes, and phenotypes. Neurology, 65(1), 87-95.

[120] Steinlechner S, et al. (2007) Co-occurrence of affective and schizophrenia spectrum disorders with PINK1 mutations. $J$ Neurol Neurosurg Psychiatry, 78(5), 532-535.

[121] Taylor TN, Greene JG, \& Miller GW (2010) Behavioral phenotyping of mouse models of Parkinson's disease. Behav Brain Res, 211(1), 1-10.

[122] Chaudhuri KR, Healy DG, \& Schapira AH (2006) Nonmotor symptoms of Parkinson's disease: Diagnosis and management. Lancet Neurol, 5(3), 235-245.

[123] Hoepken HH, et al. (2007) Mitochondrial dysfunction: Peroxidation damage and changes in glutathione metabolism in PARK6. Neurobiol Dis, 25(2), 401-411.

[124] Deng H, et al. (2008) The Parkinson's disease genes pink1 and parkin promote mitochondrial fission and/or inhibit fusion in Drosophila. Proc Natl Acad Sci U S A, 105(38), 14503-14508.

[125] Exner N, et al. (2007) Loss-of-function of human PINK1 results in mitochondrial pathology and can be rescued by parkin. J Neurosci, 27(45), 12413-12418.

[126] Sandebring A, et al. (2009) Mitochondrial alterations in PINK1 deficient cells are influenced by calcineurindependent dephosphorylation of dynamin-related protein 1 . PLoS One, 4(5), e5701.

[127] Amo T, et al. (2011) Mitochondrial membrane potential decrease caused by loss of PINK1 is not due to proton leak: But to respiratory chain defects. Neurobiol Dis, 41(1), 111-118.

[128] Morais VA, et al. (2009) Parkinson's disease mutations in PINK1 result in decreased Complex I activity and deficient synaptic function. EMBO Mol Med, 1(2), 99-111.

[129] Gandhi S, et al. (2009) PINK1-associated Parkinson's disease is caused by neuronal vulnerability to calcium-induced cell death. Mol Cell, 33(5), 627-638.

[130] Plun-Favreau H, et al. (2007) The mitochondrial protease $\mathrm{Htr} \mathrm{A} 2$ is regulated by Parkinson's disease-associated kinase PINK1. Nat Cell Biol, 9(11), 1243-1252.

[131] Um JW, et al. (2009) Molecular interaction between parkin and PINK1 in mammalian neuronal cells. Mol Cell Neurosci, 40(4), 421-432.

[132] Klinkenberg M, et al. (2010) Enhanced vulnerability of PARK6 patient skin fibroblasts to apoptosis induced by proteasomal stress. Neuroscience, 166(2), 422-434.

[133] Yuan XL, et al. (2010) R492X mutation in PTEN-induced putative kinase 1 induced cellular mitochondrial dysfunction and oxidative stress. Brain Res, 1351, 229-237.

[134] Deng $\mathrm{H}$, et al. (2005) Small interfering RNA targeting the PINK1 induces apoptosis in dopaminergic cells SH-SY5Y. Biochem Biophys Res Commun, 337(4), 11331138.
[135] Dagda RK, et al. (2009) Loss of PINK1 function promotes mitophagy through effects on oxidative stress and mitochondrial fission. J Biol Chem, 284(20), 13843-13855.

[136] Pridgeon JW, et al. (2007) PINK1 protects against oxidative stress by phosphorylating mitochondrial chaperone TRAP1. PLoS Biol, 5(7), e172-.

[137] Berthier A, et al. (2011) PINK1 displays tissue-specific subcellular location and regulates apoptosis and cell growth in breast cancer cells. Hum Pathol, 42(1), 75-87.

[138] Burke RE (2007) Inhibition of mitogen-activated protein kinase and stimulation of Akt kinase signaling pathways: Two approaches with therapeutic potential in the treatment of neurodegenerative disease. Pharmacol Ther, 114(3), 261277.

[139] Holmes A, et al. (2002) Behavioral profiles of inbred strains on novel olfactory: Spatial and emotional tests for reference memory in mice. Genes Brain Behav, 1(1), 55-69.

[140] Clark IE, et al. (2006) Drosophila pink1 is required for mitochondrial function and interacts genetically with parkin. Nature, 441(7097), 1162-1166.

[141] Park J, et al. (2006) Mitochondrial dysfunction in Drosophila PINK1 mutants is complemented by parkin. Nature, 441(7097), 1157-1161.

[142] Whitworth AJ, \& Pallanck LJ (2009) The PINK1/Parkin pathway: A mitochondrial quality control system? J Bioenerg Biomembr, 41(6), 499-503.

[143] Klein C, et al. (2011) Translational research in neurology and neuroscience 2011: Movement disorders. Arch Neurol.

[144] Jucker M (2010) The benefits and limitations of animal models for translational research in neurodegenerative diseases. Nat Med, 16(11), 1210-1214.

[145] Yang Y, et al. (2006) Mitochondrial pathology and muscle and dopaminergic neuron degeneration caused by inactivation of Drosophila Pink1 is rescued by Parkin. Proc Natl Acad Sci U S A, 103(28), 10793-10798.

[146] Bezard E, Gross CE, \& Brotchie JM (2003) Presymptomatic compensation in Parkinson's disease is not dopaminemediated. Trends Neurosci, 26(4), 215-221.

[147] Taymans JM, et al. (2007) Comparative analysis of adenoassociated viral vector serotypes $1,2,5,7$, and 8 in mouse brain. Hum Gene Ther, 18(3), 195-206.

[148] Van der Perren A, et al. (2011) Efficient and stable transduction of dopaminergic neurons in rat substantia nigra by rAAV $2 / 1,2 / 2,2 / 5,2 / 6.2,2 / 7,2 / 8$ and 2/9. Gene Ther, 18(5), 517-527.

[149] Kirik D, et al. (2002) Parkinson-like neurodegeneration induced by targeted overexpression of alpha-synuclein in the nigrostriatal system. $J$ Neurosci, 22(7), 2780-2791.

[150] Kirik D, et al. (2003) Nigrostriatal alpha-synucleinopathy induced by viral vector-mediated overexpression of human alpha-synuclein: A new primate model of Parkinson's disease. Proc Natl Acad Sci U S A, 100(5), 2884-2889.

[151] Lauwers E, et al. (2003) Neuropathology and neurodegeneration in rodent brain induced by lentiviral vector-mediated overexpression of alpha-synuclein. Brain Pathol, 13(3), 364-372.

[152] Lo Bianco C, et al. (2002) alpha -Synucleinopathy and selective dopaminergic neuron loss in a rat lentiviral-based model of Parkinson's disease. Proc Natl Acad Sci U S A, 99(16), 10813-10818.

[153] Klein RL, et al. (2002) Dopaminergic Cell Loss Induced by Human A30P alpha-Synuclein Gene Transfer to the Rat Substantia Nigra. Hum Gene Ther, 13(5), 605-612. 
[154] Van den Haute C, et al. (2003) Lentiviral vector-mediated delivery of short hairpin RNA results in persistent knockdown of gene expression in mouse brain. Hum Gene Ther, 14(18), 1799-1807.

[155] Ulusoy A, et al. (2009) Dose optimization for long-term rAAV-mediated RNA interference in the nigrostriatal projection neurons. Mol Ther, 17(9), 1574-1584.

[156] Dawson TM, Ko HS \& Dawson VL (2010) Genetic animal models of Parkinson's disease. Neuron, 66(5), 646-661.
[157] Sriram SR, et al. (2005) Familial-associated mutations differentially disrupt the solubility, localization, binding and ubiquitination properties of parkin. Hum Mol Genet, 14(17), 2571-2586.

[158] Gasser T (2009) Molecular pathogenesis of Parkinson disease: Insights from genetic studies. Expert Rev Mol Med, 11, e22. 